\title{
Population structure, dynamics and production of Hydrobia ulvae (Pennant) (Mollusca: Prosobranchia) along an eutrophication gradient in the Mondego estuary (Portugal)
}

\author{
Ana Isabel Lillebø*, Miguel Ângelo Pardal, João Carlos Marques \\ Department of Zoology, IMAR (Institute of Marine Research), University of Coimbra, 3004-517 Coimbra, Portugal. \\ * Corresponding author (fax: +351 39 23603; e-mail: ailimar@gemini.ci.uc.pt)
}

Received February 26, 1998; revised December 30, 1998; accepted February 26, 1999

\begin{abstract}
Eutrophication in the Mondego estuary gave rise to qualitative changes in the benthic community, involving the replacement of eelgrass, Zostera noltii, by green algae such as Enteromorpha spp. and Ulva sp. It seems reasonable to assume that, through time, such changes may determine a selected new trophic structure. Hydrobia ulvae, a dominant species in terms of abundance and biomass, was studied with regard to life history, population dynamics and productivity in relation to changing environmental conditions along the eutrophication gradient. The purpose was to examine to what extent this species may adapt to the new emergent conditions. During the study period, $H$. ulvae population exhibited both temporal and spatial density variations. The settlement pattern did not change along the eutrophication gradient, and took place in March, June, July and September. However, the population was denser in the less eutrophied areas, corresponding to Z. noltii meadows, when compared to the eutrophied ones, where Enteromorpha spp. blooms are usually observed. Growth was continuous through life but growth rates were higher during spring, decreased from early summer to mid fall and practically ceased during winter, and then gradually increased again up to spring. Life span was estimated as $21 \pm 3$ months. Growth productivity (P) was estimated as $93.7 \mathrm{~g} \mathrm{AFDW} \cdot \mathrm{m}^{-2} \cdot \mathrm{year}{ }^{-1}$ in the $Z$. noltii meadows, $15.2 \mathrm{~g} \mathrm{AFDW} \cdot \mathrm{m}^{-2} \cdot \mathrm{year}^{-1}$ in the eutrophied area, and $30.3 \mathrm{~g} \mathrm{AFDW} \cdot \mathrm{m}^{-2} \cdot \mathrm{year}^{-1}$ in the strongly eutrophied area. Elimination productivity (E) was estimated as $30.0 \mathrm{~g}$ AFDW $\cdot \mathrm{m}^{-2} \cdot$ year $^{-1}$ in the $Z$. noltii meadows, $51.8 \mathrm{~g} \mathrm{AFDW} \cdot \mathrm{m}^{-2} \cdot \mathrm{year}^{-1}$ in the eutrophied area, and $97.5 \mathrm{~g} \mathrm{AFDW} \cdot \mathrm{m}^{-2} \cdot \mathrm{year}^{-1}$ in the strongly eutrophied area. The average annual biomass $(\overline{\mathrm{B}})$ (standing stock) of the population was estimated as $70.2 \mathrm{~g} \mathrm{AFDW} \cdot \mathrm{m}^{-2}$ in the $\mathrm{Z}$. noltii meadows, $5.5 \mathrm{~g} \mathrm{AFDW} \cdot \mathrm{m}^{-2}$ in the eutrophied area, and $7.4 \mathrm{~g} \mathrm{AFDW} \cdot \mathrm{m}^{-2}$ in the strongly eutrophied area. $\mathrm{P} / \overline{\mathrm{B}}$ and $\mathrm{E} / \overline{\mathrm{B}}$ ratios were estimated as 1.3 and 4.8 in the $Z$. noltii meadows, 2.8 and 9.5 in the eutrophied area, and 4.5 and 13.2 in the strongly eutrophied area, respectively. As a pattern, the standing stock decreased as a function of increasing eutrophication, while $\mathrm{P} / \overline{\mathrm{B}}$ and $\mathrm{E} / \overline{\mathrm{B}}$ ratios increased following the same gradient. There is evidence that $H$. ulvae population structure and annual production are seriously affected by eutrophication, namely by macroalgal bloom dynamics. Moreover, results suggest that $H$. ulvae might be suffering a change in its adaptive strategy along the eutrophication gradient, becoming closer to a typical ' $r$ ' strategist in the strongly eutrophied areas. (C) 1999 Éditions scientifiques et médicales Elsevier SAS
\end{abstract}

Eutrophication / Hydrobia ulvae / life cycle / population dynamics / productivity

\section{INTRODUCTION}

The Mondego river drains a hydrological basin of about $6670 \mathrm{~km}^{2}$. The river estuary is the location of the Figueira da Foz mercantile harbour, which has a considerable regional importance. In addition, this estuary supports several industries, many salt-works and aquaculture farms, and also receives the nutrient and chemical discharge from agricultural areas of the lower Mondego river valley. As a whole, the estuary is under severe environmental stress, namely an ongoing eutrophication process $[18,26,29,30,32,39,40,41]$.
Benthic eutrophication has been giving rise to qualitative changes in the Mondego estuary benthic community, involving the replacement of eelgrass, Zostera noltii, by green algae such as Enteromorpha spp. and Ulva sp. while an eutrophication gradient is clearly recognisable along the south arm of the estuary (see study site). It seems reasonable to assume that such modifications will be followed by changes in the species composition and trophic structure at other levels (e.g. macrofauna composition). Through time, such changes may determine a selected new trophic structure $[32,40]$. 
This process has been monitored during the last decade, and a number of studies were carried out on the benthic communities $[5,9,26,28,29,30,31,32$, $33,39,40,41]$, in which Hydrobia ulvae was identified as a dominant species in Z. noltii meadows, exhibiting a stable population and significant biomass all year. Moreover, this species was found along the eutrophication gradient, up to the inner areas of the estuary, showing a clear dependence on the occurrence of green macroalgae. Therefore, it was important to study the life history, population dynamics and production of $H$. ulvae in relation to changing environmental conditions along the eutrophication gradient. The purpose was to determine to what extent this species may adapt to the new emergent conditions in the south arm of the Mondego estuary.

\section{MATERIALS AND METHODS}

\subsection{Study site}

The Mondego estuary is a warm-temperate coastal system on the western coast of Portugal (figure 1). It consists of two arms, north and south, with very different hydrologic characteristics. The northern arm is deeper, while the southern arm is largely silted up, especially in upstream areas, which causes most of the freshwater discharge to flow through the northern arm. Consequently, the water circulation in the southern arm is mainly dependent on tidal activity and on the Usually small freshwater input of a tributary, the Pranto river, which is controlled by a sluice. Furthermore, due to differences in depth, the tidal penetration

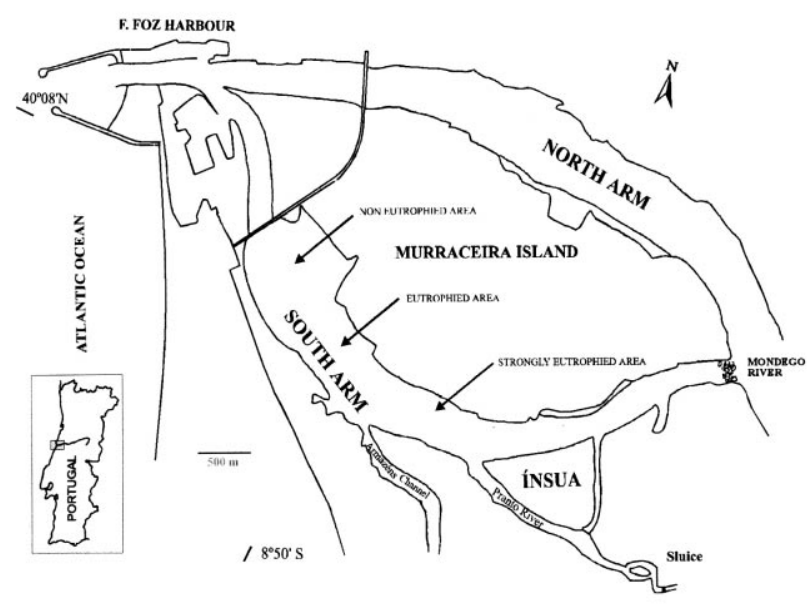

Figure 1. The Mondego estuary with indication of the eutrophication gradient in the south arm. is faster in the northern arm, causing large daily salinity fluctuations, while daily temperature changes are more pronounced in the southern arm [28].

Although, a large part of the southern arm intertidal area still remains more or less unchanged, exhibiting sand muddy bottoms covered by Spartina maritima marshes [27] and Zostera noltii meadows, macroalgal blooms of Enteromorpha spp. have been regularly observed during the last 15 years. This is probably a result of excessive nutrient release into the estuary, coupled with longer persistence of nutrients (nitrogen and phosphorous) in the water column [18, 26, 32, 40]. Such macroalgal blooms may not occur in exceptionally rainy years due to the occurrence of low salinity values for long periods as a result of the Pranto river discharge $[34,40]$.

\subsection{Field programme}

The benthic communities were sampled fortnightly from March 1993 to March 1994, along the eutrophication gradient, during low tide. The gradient consisted in three different sites, the Z. noltii community, a eutrophied area and a strongly eutrophied area, from where macrophytes disappeared while Enteromorpha blooms occur each year.

As a general trend, Enteromorpha biomass increases from early winter (February/March) up to July, when an algal crash usually occurs. After the summer, a second smaller biomass peak may normally be observed in September followed by a decrease in winter [40].

At each station, ten random samples were taken using a manual core, each replicate corresponding to $141 \mathrm{~cm}^{2}$ and approximately $3 \mathrm{~L}$ sediment. Samples were preserved in $4 \%$ buffered formalin, and later sieved through a $0.5-\mathrm{mm}$ mesh size sieve. Animals were separated and kept in $70 \%$ ethanol.

At each station and sampling date, salinity, temperature, $\mathrm{pH}$ and dissolved oxygen were measured in situ in low water pools. Sediment samples were also collected and subsequently analysed for organic matter content and granulometry. The organic matter was determined by loss on ignitions $\left(8 \mathrm{~h}\right.$ at $\left.450{ }^{\circ} \mathrm{C}\right)$, for each sampling date, and the granulometry was analysed seasonally and classified according to the nomenclature proposed by Brown and McLachan [8].

\subsection{Laboratory procedures}

Hydrobia ulvae individuals were counted and their shell height measured to the nearest $0.1 \mathrm{~mm}$. As a number of individuals had damaged shells, we determined other measures, such as the maximum width and maximum length unto the last spire, which were then converted to shell height. These conversions were 
$\mathbf{A}$

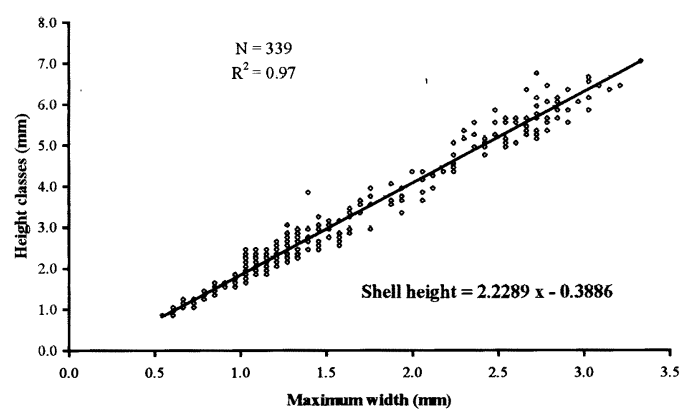

B
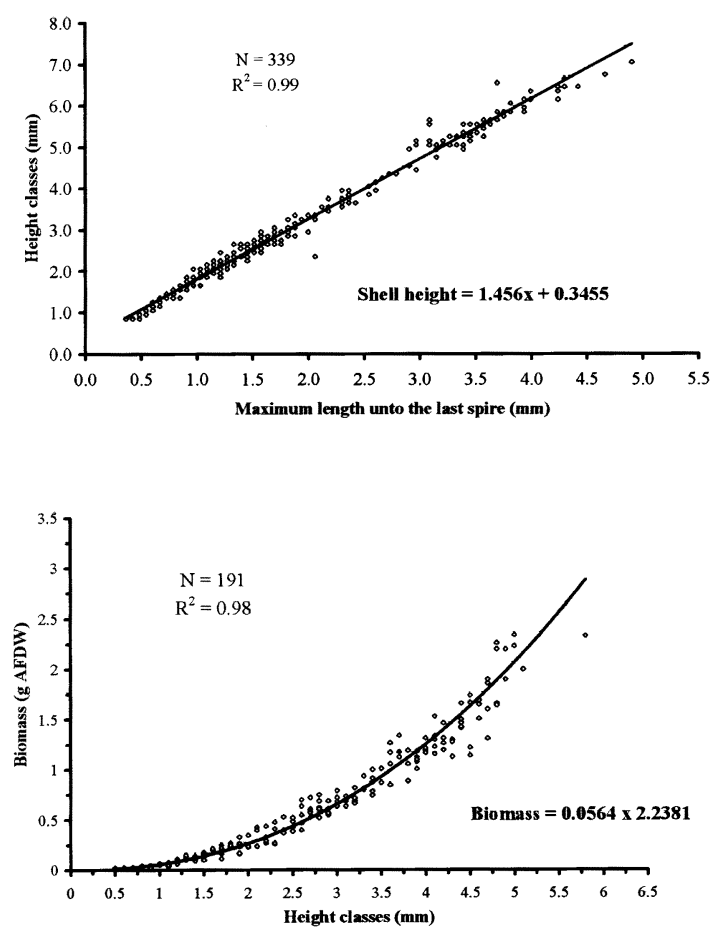

Figure 2. Hydrobia ulvae biometric relationships.

based on the biometrics relationships of 339 individuals with intact shells (figure $2 A, B$ ). The relationship between total length and ash free dry weight was also established and used in production estimates. There were not any significant differences between equations established for each season based on analysis of variance $(P=0.05)$. Therefore, the data set of 191 individuals measured and weighed throughout the study was used to provide a single regression equation (figure $2 \mathrm{C}$ ). Individuals were dried at $60{ }^{\circ} \mathrm{C}$ for $72 \mathrm{~h}$ and weighed to the nearest $0.01 \mathrm{mg}$. Ash free dry weight (AFDW) was assessed after combustion of the samples for $8 \mathrm{~h}$ at $450{ }^{\circ} \mathrm{C}$.

\subsection{Data analysis}

We analysed the population spatial distribution by using the index $I=S^{2} / \bar{X}[14]$.

Multiple regression models were developed to relate the variation of $H$. ulvae biomass with salinity, temperature, dissolved oxygen, organic matter content in sediments, and macroalgae and macrophytes biomass. The fitted regression models were expressed as:

$$
Y^{\prime}=a+b_{1} X_{1}+b_{2} X_{2}+\ldots+b_{k} X_{k}
$$

where $Y^{\prime}$ is the values of a given dependent variable (e.g. biomass) predicted by the equation, $X_{1}, X_{2} \ldots X_{k}$ independent variables (e.g. salinity).

The solutions are the estimate of the regression coefficients $a, b_{1}, b_{2} \ldots b_{k}$. The significance of fitted regressions was tested by using analysis of variance $(\mathrm{F})$, and the $t$-test for the regression coefficients, as described in Edwards [12, 13]. Regressions were estimated using the Stepwise Forward Analysis method performed with the STATGRAPHICS 4.0 statistical package.

Growth rates were estimated by tracking recognisable cohorts along size-frequency distributions (0.02-mm classes) from successive sample dates. Sizefrequency analysis was performed using the ANAMOD software package [38], in which the analysis reliability was tested using the $\mathrm{X}^{2}$ and G-tests $(P=0.05)$.

Growth rates are usually not constant throughout the year. Therefore, we used the following model proposed by Gaschütz et al. [19] to express growth taking into account seasonal variations:

$$
\mathrm{L}_{\mathrm{t}}=\mathrm{L}_{\infty}\left\{1-\mathrm{e}^{-[\mathrm{KD}(\mathrm{t}-\mathrm{to})+\mathrm{C}(\mathrm{kD} / 2 \Pi) \sin 2 \Pi(\mathrm{t}-\mathrm{ts})]}\right\}^{1 / \mathrm{D}}
$$

where $L_{t}$ is the length of the organism at a given moment $t, L_{\infty}$ the maximum possible length of the organism, $t$ the given instant, $t_{0}$ the instant when the organism would have a length $=0, t_{\mathrm{s}}$ the time interval between growth start (when $\mathrm{t}=0$ ) and the first growth oscillation, growth is expressed by a sin curve with a 1-year period, $\mathrm{K}$ the intrinsic growth rate, $\mathrm{C}$ the parameter in which the values can range from 0 to 1 depending on the species, and D the parameter that expresses metabolic deviations from von Bertalanffy's $2 / 3$ rule (the metabolism of an organism is proportional to his weight by $\mathrm{a}^{2 / 3}$ power).

We estimated both growth (P) and elimination (E) production as derived by Allen [1]. Values of $\mathrm{P}$ and $\mathrm{E}$ for each cohort for a given time interval are expressed as:

$$
\begin{aligned}
& \mathrm{P}=\left[\left(\mathrm{N}_{\mathrm{t}}+\mathrm{N}_{\mathrm{t}+1}\right) / 2\right]\left(\bar{W}_{\mathrm{t}+1}-\bar{W}_{\mathrm{t}}\right) \text { for } \bar{W}_{\mathrm{t}+1}>\bar{W}_{\mathrm{t}} \\
& \mathrm{E}=\left[\left(\bar{W}_{\mathrm{t}}+\bar{W}_{\mathrm{t}+1}\right) / 2\right]\left(\mathrm{N}_{\mathrm{t}}-\mathrm{N}_{\mathrm{t}+1}\right) \text { for } \mathrm{N}_{\mathrm{t}}>\mathrm{N}_{\mathrm{t}+1}
\end{aligned}
$$


where $\mathrm{N}$ is the number of individuals from a cohort at each sample date, $\bar{W}$ the mean individual biomass for each sample date, $\mathrm{t}$ and $\mathrm{t}+1$ the consecutive sampling dates.

Total values of $\mathrm{P}$ and $\mathrm{E}$ for each cohort are expressed as:

$$
\begin{aligned}
P & =\sum_{t=0}^{t=n}\left[\left(\mathrm{~N}_{\mathrm{t}}+\mathrm{N}_{\mathrm{t}+1}\right) / 2\right] \Delta \bar{W} \\
\mathrm{E} & =\sum_{t=0}^{t=n}\left[\left(\bar{W}_{\mathrm{t}}+\bar{W}_{\mathrm{t}+1}\right) / 2\right] \Delta \mathrm{N}
\end{aligned}
$$

Total values of $\mathrm{P}$ and $\mathrm{E}$, for the population, are expressed as:

$$
P=\sum_{n=1}^{N} \mathrm{P}_{\mathrm{cn}} \quad \text { and } \quad \mathrm{E}=\sum_{n=1}^{N} \mathrm{E}_{\mathrm{cn}}
$$

where $\mathrm{P}_{\mathrm{cn}}$ and $\mathrm{E}_{\mathrm{cn}}$ are the growth and elimination production of the cohort $n . \mathrm{P} / \overline{\mathrm{B}}$ and $\mathrm{E} / \overline{\mathrm{B}}$ ratios were determined. $\overline{\mathrm{B}}$ (mean population biomass) is expressed as:

$$
\overline{\mathrm{B}}=(1 / \mathrm{T}) \sum_{n=1}^{N}\left(\overline{\mathrm{B}}_{\mathrm{n}} \times \mathrm{t}\right)
$$

where T is the period of study, $N$ the number of cohorts in the period $\mathrm{T}, \overline{\mathrm{B}}_{\mathrm{n}}$ the mean biomass of the cohort $n$ and $\mathrm{t}$ the duration of the cohort $n$.

\section{RESULTS}

\subsection{Macrophytes and macroalge biomass}

Z. noltii biomass exhibited a seasonal variation in biomass. During fall and winter, total biomass con-

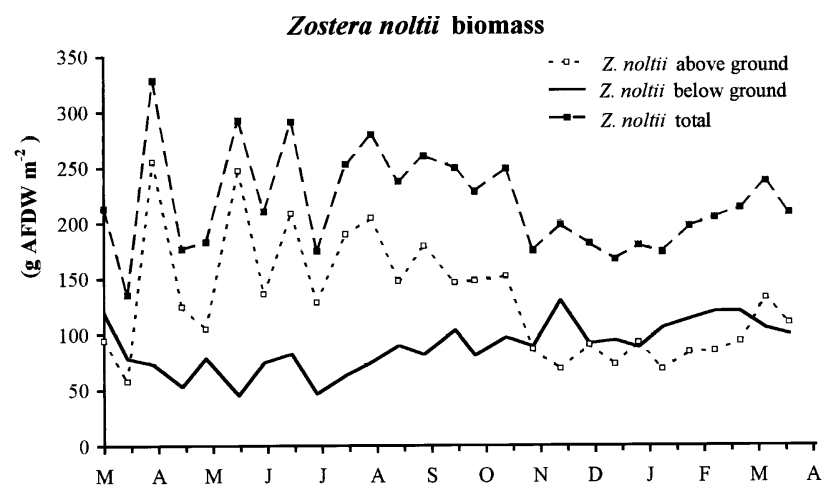

Figure 3. Zostera noltii biomass in the south arm of the Mondego estuary between March 93 and March 94 . sisted essentially of rhizomes, and during spring and summer, total biomass increased due to the growth of shoots (figure 3).

The composition of macroalgal biomass changed along the eutrophication gradient. Red macroalgae, essentially Gracilaria sp., were relatively abundant at the Z. noltii meadows, the non-eutrophied area, decreasing along the eutrophication gradient while green macroalgae showed exactly the opposite pattern (figure 4). In the strongly eutrophied area, green macroalgae presented a typical spring bloom, with Enteromor-
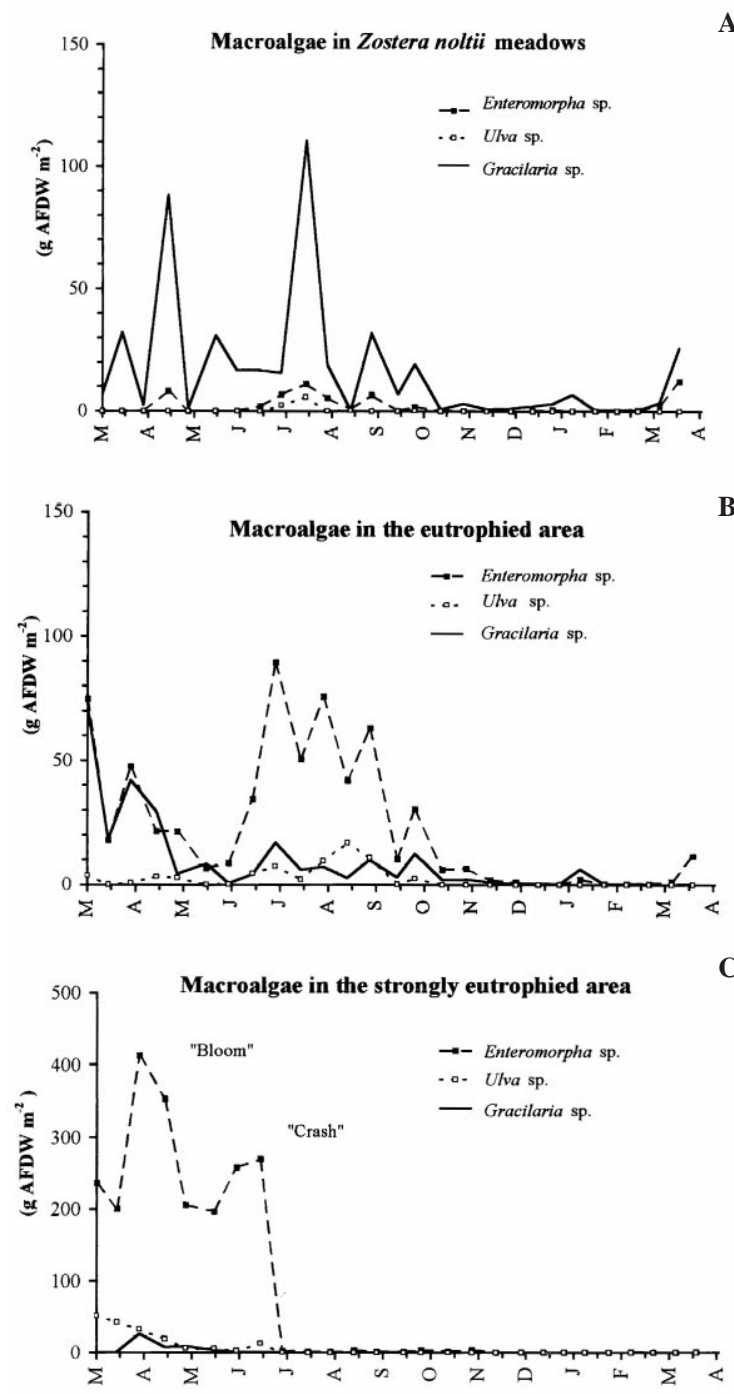

Figure 4. Macroalgal biomass in the south arm of the Mondego estuary between March 93 and March 94. A, Zostera noltii meadows; $\mathrm{B}$, eutrophied area; $\mathrm{C}$, strongly eutrophied area. 


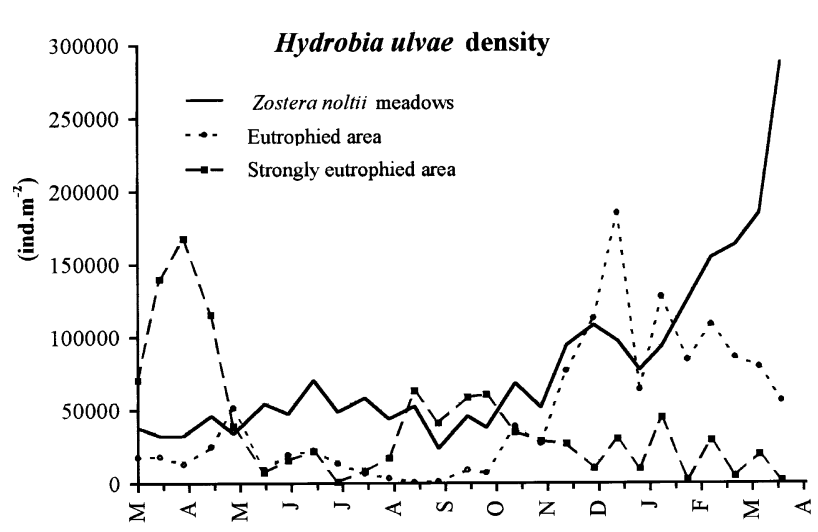

Figure 5. Abundance of Hydrobia ulvae along an eutrophication gradient, in the south arm of the Mondego estuary between March 93 and March 94.

pha spp. biomass reaching $413.19 \mathrm{~g} \mathrm{AFDW} \cdot \mathrm{m}^{-2}$ (April 7, 1993), followed by an algal crash in early summer (July 6, 1993) (figure 4C). However, no algal bloom was observed in spring 1994 due to the fact that this was a particularly rainy year.

\subsection{Abundance}

Population density changed throughout the period of study but the pattern of variation was not the same at the three sampling stations (figure 5). Density was consistently higher at the $Z$. noltii meadows, increasing gradually from November 1993 up to a maximum during spring in 1994 (approximately 288000 ind $\cdot \mathrm{m}^{-2}$, March 23, 1994). The population density increased during fall and winter in the eutrophied area, reaching a maximum of approximately 151000 ind $\cdot \mathrm{m}^{-2}$ (December 17, 1993). Then, it de- creased again through spring, although not reaching the exceptionally low value of $657 \mathrm{ind} \cdot \mathrm{m}^{-2}$ recorded in the previous year (August 20, 1993). In the strongly eutrophied area, population density reached a maximum of approximately $167000 \mathrm{ind} \cdot \mathrm{m}^{-2}$ (April 7, 1993), during the algal bloom, and a minimum of 699 ind $\cdot \mathrm{m}^{-2}$ (July 6, 1993) after the algal crash (figure 5). After the algal crash, the population recovered to a certain extent, but without reaching the previous density levels.

\subsection{Environmental factors}

Silt and clay were the main constituents of sediments at the Z. noltii meadows, while in areas covered with algae, sediments mainly consisted of fine sand (table I). Other environmental factors were also determined fortnightly at each sampling station (tables II, III) and taken into account in multiple regression analysis.

In the $Z$. noltii meadows, $H$. ulvae biomass (g AFDW) was positively correlated with green algae (Enteromorpha spp. + Ulva sp.), biomass $(\log +1)$ and negatively correlated with salinity and $Z$. noltii belowgrownd biomass; $\left(P=0.000 ; r^{-2}=0.70\right)$

H. ulvae biomass $=105+15.0(\ln +1$ green algal $)$ -1.38 (Sal.) -0.282 (Z. below-ground)

Regarding the strongly eutrophied area, we analysed separately the algal bloom and post-algal crash scenarios (figure 6). During the algal bloom, H. ulvae biomass appeared positively correlated with green algae biomass, with organic matter content in sediments, and with dissolved oxygen, $(P=0.007$; $\left.r^{-2}=0.94\right)$ :

H. ulvae biomass $=-74.2+0.0333$ (green algae) +0.150 (org. mat.) $+1.89\left(\mathrm{O}_{2}\right)$

After the algal crash, $H$. ulvae biomass appeared positively correlated with green algae biomass (En-

Table I. Relative frequency of sediment granulometry classes determined seasonally along the eutrophication gradient in the south arm of the Mondego estuary.

\begin{tabular}{|c|c|c|c|c|c|c|c|c|c|}
\hline \multirow[b]{2}{*}{ Classes (mm) } & \multicolumn{3}{|c|}{ Zostera noltii meadows } & \multicolumn{3}{|c|}{ Eutrophied area } & \multicolumn{3}{|c|}{ Strongly eutrophied area } \\
\hline & April & June & Dec. & April & June & Dec. & April & June & Dec. \\
\hline$>2$ & 0.94 & 0.29 & 3.86 & 0.46 & 0.21 & 0.74 & 0.42 & 0.11 & 0.03 \\
\hline$>1,<2$ & 1.03 & 1.10 & 1.44 & 0.42 & 0.31 & 0.35 & 0.62 & 0.57 & 0.33 \\
\hline$>0.500,<1.0$ & 4.28 & 1.36 & 2.27 & 1.72 & 0.63 & 1.20 & 2.06 & 1.72 & 1.34 \\
\hline$>0.250,<0.500$ & 1.11 & 14.79 & 16.23 & 6.94 & 2.50 & 16.21 & 7.71 & 10.78 & 15.08 \\
\hline$>0.125,<0.250$ & 22.35 & 17.21 & 19.99 & 24.66 & 21.75 & 47.23 & 36.86 & 33.13 & 40.02 \\
\hline$>0.063,<0.125$ & 63.10 & 19.48 & 19.30 & 59.49 & 25.14 & 21.22 & 49.43 & 28.12 & 26.19 \\
\hline$>0.038,<0.063$ & 4.54 & 31.34 & 27.85 & 3.68 & 35.11 & 7.56 & 1.80 & 20.45 & 9.87 \\
\hline$<0.038$ & 2.65 & 14.43 & 9.06 & 2.63 & 14.35 & 5.49 & 1.10 & 5.12 & 7.14 \\
\hline
\end{tabular}


Table II. Hydrobia ulvae biomass and environmental factors taken into account in multiple regression analysis in Zostera noltii meadows.

\begin{tabular}{|c|c|c|c|c|c|c|c|c|c|c|}
\hline Date & $\begin{array}{c}\text { H. ulvae } \\
\left.\text { (g AFDW. }{ }^{-2}\right)\end{array}$ & $\begin{array}{c}\text { Temp. } \\
\left({ }^{\circ} \mathrm{C}\right)\end{array}$ & $\begin{array}{c}\mathrm{O}_{2} \\
\left(\mathrm{mg} \cdot \mathrm{L}^{-1}\right)\end{array}$ & $\begin{array}{l}\text { Salinity } \\
\left(\mathrm{g} \cdot \mathrm{L}^{-1}\right)\end{array}$ & $\begin{array}{l}\text { Org. matter } \\
\left(\mathrm{g} \cdot \mathrm{m}^{-2}\right)\end{array}$ & $\begin{array}{c}\text { Green algae } \\
\left(\mathrm{g} \text { AFDW } \cdot \mathrm{m}^{-2}\right)\end{array}$ & $\begin{array}{c}\text { Red algae } \\
\left(\mathrm{g} \text { AFDW } \cdot \mathrm{m}^{-2}\right)\end{array}$ & $\begin{array}{l}\text { Z. noltii above-ground } \\
\qquad\left(\mathrm{g} \text { AFDW } \cdot \mathrm{m}^{-2}\right)\end{array}$ & $\begin{array}{l}\text { Z. noltii below-ground } \\
\qquad\left(\mathrm{g} \mathrm{AFDW} \cdot \mathrm{m}^{-2}\right)\end{array}$ & $\begin{array}{l}\text { Z. noltii total } \\
\left(\mathrm{g} \text { AFDW } \cdot \mathrm{m}^{-2}\right)\end{array}$ \\
\hline 24-Mar & 47.71 & 15.1 & 12.7 & 22.0 & 558.10 & 0.01 & 19.58 & 75.63 & 98.62 & 212.06 \\
\hline 7-Apr & 47.59 & 15.6 & 11.8 & 24.0 & 551.06 & 0.01 & 17.41 & 156.21 & 75.63 & 347.75 \\
\hline 23-Apr & 58.67 & 17.5 & 11.3 & 32.5 & 501.06 & 4.16 & 45.45 & 189.76 & 62.89 & 425.64 \\
\hline 6-Маy & 52.10 & 17.9 & 11.9 & 29.7 & 359.97 & 4.19 & 44.84 & 114.44 & 65.57 & 332.11 \\
\hline 24-May & 56.91 & 17.7 & 12.5 & 25.7 & 351.08 & 0.03 & 16.13 & 175.79 & 62.01 & 382.91 \\
\hline 7-Jun & 54.46 & 22.4 & 15.6 & 24.5 & 534.39 & 0.11 & 23.79 & 191.42 & 60.09 & 435.12 \\
\hline 22-Jun & 51.40 & 23.6 & 14.7 & 28.5 & 516.25 & 1.10 & 16.65 & 172.19 & 78.76 & 432.76 \\
\hline 6-Jul & 55.82 & 23.7 & 10.9 & 32.2 & 502.17 & 5.59 & 16.06 & 168.44 & 64.70 & 403.46 \\
\hline 22-Jul & 60.81 & 25.4 & 11.5 & 33.4 & 481.44 & 13.04 & 63.06 & 159.02 & 54.86 & 377.61 \\
\hline 5-Aug & 58.84 & 25.7 & 10.3 & 32.4 & 491.81 & 11.14 & 64.73 & 197.10 & 69.05 & 444.21 \\
\hline 20-Aug & 49.55 & 23.9 & 7.9 & 29.8 & 538.84 & 3.21 & 9.68 & 175.92 & 82.54 & 444.98 \\
\hline 3-Sep & 48.39 & 21.0 & 8.1 & 29.0 & 499.95 & 3.80 & 16.18 & 163.20 & 85.74 & 418.50 \\
\hline 20-Sep & 45.49 & 23.0 & 8.5 & 28.8 & 515.88 & 3.38 & 19.40 & 162.59 & 92.54 & 418.02 \\
\hline 1-Oct & 43.20 & 23.2 & 11.3 & 29.6 & 572.54 & 1.18 & 13.14 & 146.83 & 92.26 & 393.80 \\
\hline 18-Oct & 47.30 & 19.2 & 11.4 & 20.8 & 483.66 & 1.21 & 10.06 & 149.59 & 89.05 & 386.84 \\
\hline 2-Nov & 49.90 & 16.7 & 9.1 & 15.6 & 504.77 & 0.23 & 1.95 & 118.88 & 93.18 & 346.30 \\
\hline 17-Nov & 45.90 & 15.0 & 13.4 & 18.8 & 522.54 & 0.20 & 1.94 & 77.35 & 109.53 & 285.00 \\
\hline 3-Dec & 58.23 & 14.2 & 14.7 & 16.0 & 489.58 & 0.14 & 1.02 & 79.39 & 110.78 & 268.54 \\
\hline 17-Dec & 71.90 & 14.2 & 11.0 & 15.0 & 508.84 & 0.06 & 1.64 & 81.49 & 93.07 & 255.00 \\
\hline 30-Dec & 67.25 & 14.2 & 8.1 & 16.8 & 529.21 & 0.34 & 2.53 & 82.44 & 91.27 & 255.67 \\
\hline 12-Jan & 61.49 & 13.2 & 7.4 & 11.8 & 580.69 & 0.33 & 4.85 & 80.02 & 96.80 & 258.05 \\
\hline 27-Jan & 58.77 & 9.5 & 9.1 & 8.4 & 562.91 & 0.12 & 3.57 & 75.75 & 109.55 & 263.18 \\
\hline $10-\mathrm{Feb}$ & 61.98 & 6.3 & 10.2 & 12.9 & 502.55 & 0.11 & 0.65 & 84.11 & 116.88 & 280.91 \\
\hline 24-Feb & 70.82 & 9.2 & 9.1 & 10.4 & 435.51 & 0.26 & 0.66 & 88.96 & 120.36 & 295.85 \\
\hline 10-Mar & 72.80 & 15.7 & 7.0 & 6.3 & 424.03 & 1.58 & 2.00 & 112.77 & 112.88 & 326.52 \\
\hline 23-Mar & 73.72 & 19.1 & 12.5 & 13.1 & 445.51 & 7.43 & 14.75 & 121.04 & 102.46 & 340.41 \\
\hline
\end{tabular}

teromorpha spp. + Ulva sp.; $\ln +1)$ and dissolved oxygen $\left(P=0.001 ; r^{-2}=0.63\right)$ :

H. ulvae biomass $=-53.6+27.0(\ln +1$ green algae $)$ $+4.718\left(\mathrm{O}_{2}\right)$

\subsection{Growth and life span}

Size-frequency polymodal distributions were analysed for $H$. ulvae recognisable cohorts (figure 7). Significant differences in the population structure along the eutrophication gradient were observed. Individuals reached larger dimensions at the $Z$. noltii meadows when compared to macroalgae-covered areas. Nevertheless, the same settlement pattern was observed along the eutrophication gradient, respectively in March, June, July and September $\left(X^{2}\right.$ and $G$ not significant, $P \leq 0.05)$. New cohorts were detected with an average shell height of $1.11 \mathrm{~mm}$ at the $Z$. noltii meadows, $1.07 \mathrm{~mm}$ at the eutrophied area, and $1.10 \mathrm{~mm}$ at the strongly eutrophied area.

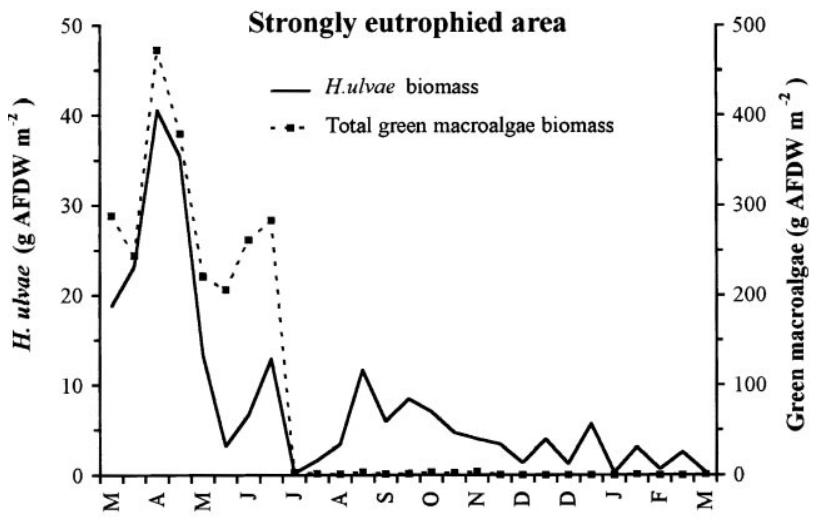

Figure 6. Green macroalgal biomass and Hydrobia ulvae abundance variations in the strongly eutrophied area in the south arm of the Mondego estuary between March 93 and March 94. 
Table III. Hydrobia ulvae biomass and environmental factors taken into account in multiple regression analysis in strongly eutrophied area.

\begin{tabular}{|c|c|c|c|c|c|c|c|}
\hline Date & $\begin{array}{c}\text { H. ulvae } \\
\left(\mathrm{g} \mathrm{AFDW} \cdot \mathrm{m}^{-2}\right)\end{array}$ & $\begin{array}{l}\text { Temp. } \\
\left({ }^{\circ} \mathrm{C}\right)\end{array}$ & $\begin{array}{c}\mathrm{O}_{2} \\
\left(\mathrm{mg} \cdot \mathrm{L}^{-1}\right)\end{array}$ & $\begin{array}{l}\text { Salinity } \\
\left(\mathrm{g} \cdot \mathrm{L}^{-1}\right)\end{array}$ & $\begin{array}{l}\text { Org. matter } \\
\left(\mathrm{g} \cdot \mathrm{m}^{-2}\right)\end{array}$ & $\begin{array}{c}\text { Green algae } \\
\left(\mathrm{g} \mathrm{AFDW} \cdot \mathrm{m}^{-2}\right)\end{array}$ & $\begin{array}{c}\text { Red algae } \\
\left(\mathrm{g} \mathrm{AFDW} \cdot \mathrm{m}^{-2}\right)\end{array}$ \\
\hline 24-Mar & 20.64 & 20.5 & 20.0 & 22.2 & 304.44 & 265.07 & 0.88 \\
\hline 7-Apr & 31.52 & 21.8 & 19.4 & 22.0 & 387.69 & 344.11 & 13.74 \\
\hline 23-Apr & 36.23 & 19.7 & 14.2 & 29.5 & 446.86 & 408.85 & 16.99 \\
\hline 6-May & 22.63 & 17.2 & 12.6 & 23.5 & 444.82 & 291.73 & 8.32 \\
\hline 24-May & 8.27 & 18.0 & 12.5 & 21.5 & 344.43 & 207.05 & 6.33 \\
\hline 7-Jun & 4.95 & 24.7 & 147.4 & 25.5 & 327.29 & 231.55 & 2.26 \\
\hline 22-Jun & 9.82 & 30.2 & 13.3 & 29.5 & 337.49 & 271.62 & 0.56 \\
\hline 6-Jul & 6.58 & 30.8 & 9.6 & 29.5 & 362.38 & 142.60 & 0.43 \\
\hline 22-Jul & 0.94 & 32.4 & 9.4 & 27.4 & 343.61 & 2.05 & 0.29 \\
\hline 5-Aug & 2.52 & 30.4 & 8.7 & 29.7 & 353.00 & 1.34 & 0.09 \\
\hline 20-Aug & 7.53 & 28.1 & 7.6 & 28.1 & 399.93 & 2.33 & 0.15 \\
\hline 3-Sep & 8.81 & 27.6 & 8.1 & 27.9 & 332.19 & 2.30 & 0.19 \\
\hline 20-Sep & 9.26 & 27.3 & 8.0 & 28.4 & 326.88 & 1.06 & 0.53 \\
\hline 1-Oct & 48.58 & 23.6 & 10.2 & 29.5 & 404.83 & 2.25 & 0.54 \\
\hline 18 -Oct & 74.38 & 21.9 & 13.0 & 20.4 & 315.05 & 2.79 & 0.12 \\
\hline 2-Nov & 52.15 & 21.1 & 13.8 & 13.8 & 302.80 & 2.85 & 0.14 \\
\hline 17-Nov & 22.05 & 19.3 & 15.1 & 17.0 & 321.17 & 1.86 & 0.15 \\
\hline 3-Dec & 2.78 & 19.2 & 12.9 & 14.7 & 307.70 & 0.07 & 0.08 \\
\hline 17-Dec & 2.93 & 17.7 & 10.7 & 17.0 & 300.76 & 0.08 & 0.10 \\
\hline 30-Dec & 3.01 & 16.7 & 11.3 & 19.5 & 288.93 & 0.07 & 0.09 \\
\hline 12-Jan & 3.72 & 15.3 & 11.7 & 11.6 & 319.13 & 0.02 & 0.03 \\
\hline 27-Jan & 2.99 & 12.5 & 10.9 & 6.6 & 259.14 & 0.01 & 0.00 \\
\hline $10-\mathrm{Feb}$ & 1.71 & 10.5 & 11.0 & 10.6 & 222.82 & 0.47 & 0.00 \\
\hline 24-Feb & 1.87 & 11.7 & 9.0 & 9.5 & 265.26 & 0.50 & 0.00 \\
\hline 10-Mar & 1.60 & 15.7 & 8.9 & 8.1 & 257.91 & 0.08 & 0.05 \\
\hline
\end{tabular}

Growth was continuous through life (figure 8). Nevertheless, growth rates were highest in spring, decreased from early summer to mid fall, practically ceased in winter (figure 9), and gradually increased again up to spring. Lower growth rates during winter were probably a result of lower temperatures. During the rest of the year, higher growth rates were probably a function of higher temperatures as well as increased food resources.

Life span was estimated at $21 \pm 3$ months. Individuals presented a mean shell height between $3.78 \mathrm{~mm}$ $(\mathrm{SD} \pm 0.30)$ and $4.75 \mathrm{~mm}(\mathrm{SD} \pm 0.43)$ after the first year, and $5.84 \mathrm{~mm}(\mathrm{SD} \pm 0.82)$ after 21 months. Data on cohort $\mathrm{C} 5$ at the $Z$. noltii meadows were used to calibrate a growth model (figure 9). The model parameters were estimated as follows: $\mathrm{L}_{\infty}=7.95 \mathrm{~mm}$, corresponding to the largest individual observed; $\mathrm{K}=0.729 ; \mathrm{t}_{0}=-0.112 ; \mathrm{t}_{\mathrm{s}}=-0.111 ; \mathrm{C}=0.529 ; \mathrm{D}=1$, since it was assumed that there were no deviations from von Bertalanffy's $2 / 3$ rule. Unfortunately, since the H. ulvae life span is longer than our study period
(377 d), we could not take into account a second settlement in order to validate the model.

\subsection{Life cycle}

The reproductive period was studied taking into account the occurrence of egg masses, which are fixed on live shells of its congeners [17]. We found a very low percentage of the population carrying egg masses and therefore data from the three sampling stations were pooled together for the analysis (figure 10). Two main reproductive peaks are recognisable in the Mondego estuary population, in spring and in late summer.

\subsection{Production estimates}

Relationships between height and ash free dry weight (g AFDW) (figure 2C) were used to estimate production taking into account cohort growth and mortality. Growth productivity (P) was estimated at $93.7 \mathrm{~g} \mathrm{AFDW} \cdot \mathrm{m}^{-2} \cdot$ year $^{-1}$ in the $Z$. noltii meadows, 
Zostera noltii meadows

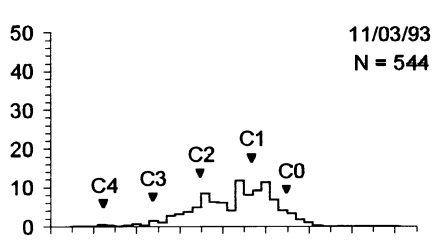

$\begin{array}{lllllllll}0.0 & 1.0 & 2.0 & 3.0 & 4.0 & 5.0 & 6.0 & 7.0 & 8.0\end{array}$

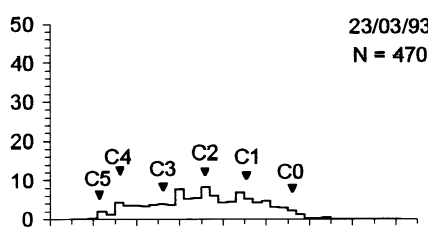

$\begin{array}{lllllllll}0.0 & 1.0 & 2.0 & 3.0 & 4.0 & 5.0 & 6.0 & 7.0 & 8.0\end{array}$
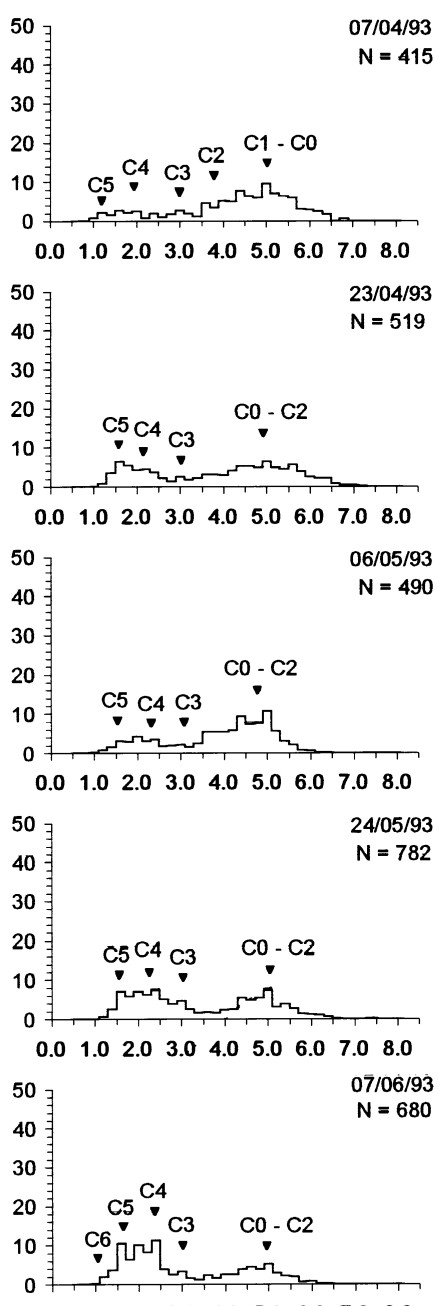

$\begin{array}{lllllllll}0.0 & 1.0 & 2.0 & 3.0 & 4.0 & 5.0 & 6.0 & 7.0 & 8.0\end{array}$

Shell height (mm)
Eutrophied area

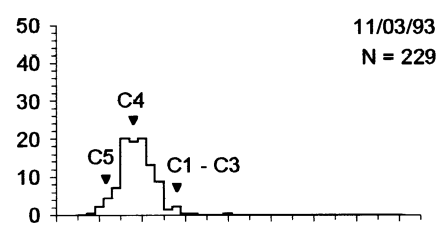

$\begin{array}{llllllllll}0.0 & 1.0 & 2.0 & 3.0 & 4.0 & 5.0 & 6.0 & 7.0 & 8.0\end{array}$

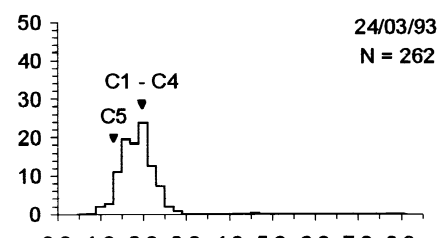

$\begin{array}{lllllllll}0.0 & 1.0 & 2.0 & 3.0 & 4.0 & 5.0 & 6.0 & 7.0 & 8.0\end{array}$
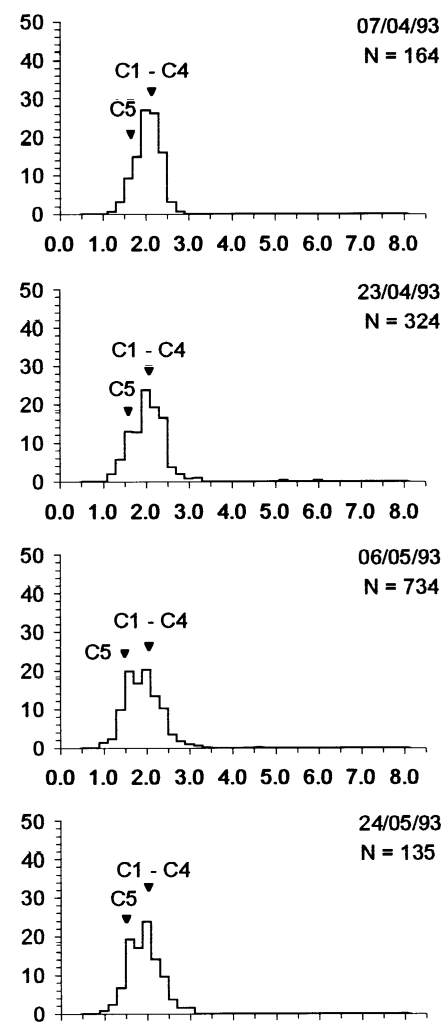

$\begin{array}{lllllllll}0.0 & 1.0 & 2.0 & 3.0 & 4.0 & 5.0 & 6.0 & 7.0 & 8.0\end{array}$

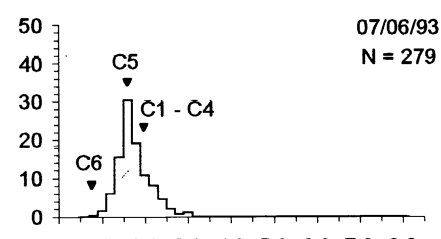

$\begin{array}{lllllllll}0.0 & 1.0 & 2.0 & 3.0 & 4.0 & 5.0 & 6.0 & 7.0 & 8.0\end{array}$

Shell height (mm)
Strongly eutrophied area

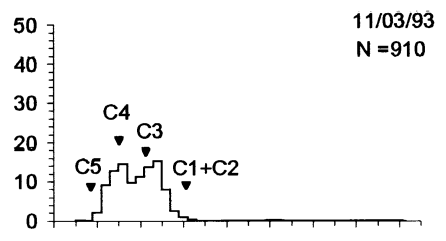

$\begin{array}{lllllllll}0.0 & 1.0 & 2.0 & 3.0 & 4.0 & 5.0 & 6.0 & 7.0 & 8.0\end{array}$

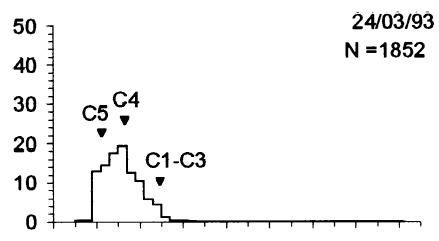

$\begin{array}{lllllllllll}0.0 & 1.0 & 2.0 & 3.0 & 4.0 & 5.0 & 6.0 & 7.0 & 8.0\end{array}$
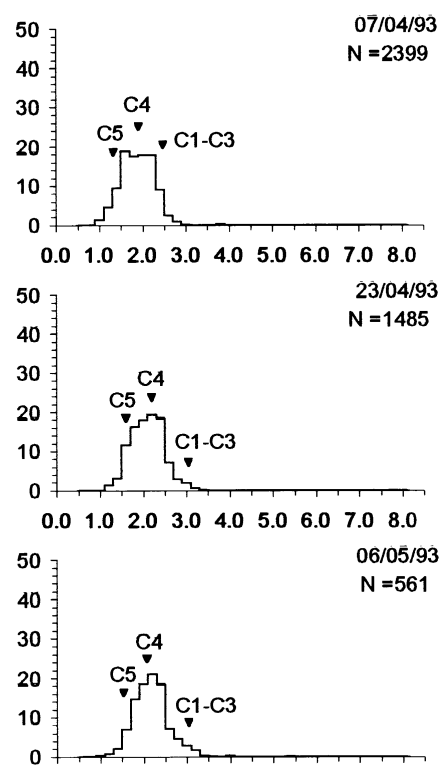

$\begin{array}{lllllllll}0.0 & 1.0 & 2.0 & 3.0 & 4.0 & 5.0 & 6.0 & 7.0 & 8.0\end{array}$

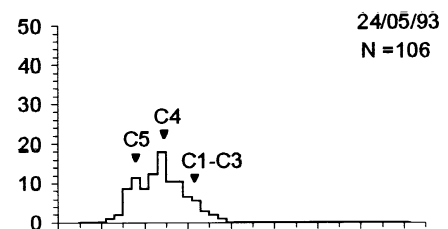

$\begin{array}{lllllllll}0.0 & 1.0 & 2.0 & 3.0 & 4.0 & 5.0 & 6.0 & 7.0 & 8.0\end{array}$

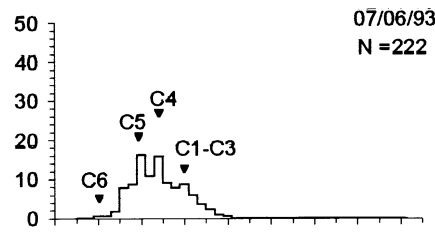

$\begin{array}{lllllllll}0.0 & 1.0 & 2.0 & 3.0 & 4.0 & 5.0 & 6.0 & 7.0 & 8.0\end{array}$

Shell height $(\mathrm{mm})$

Figure 7. Size-frequency polymodal distribution of Hydrobia ulvae, in the south arm of the Mondego estuary, along an eutrophication gradient between March 93 and March 94. Sampling dates are indicated. N, Number of individuals analysed. 
Zostera noltii meadows
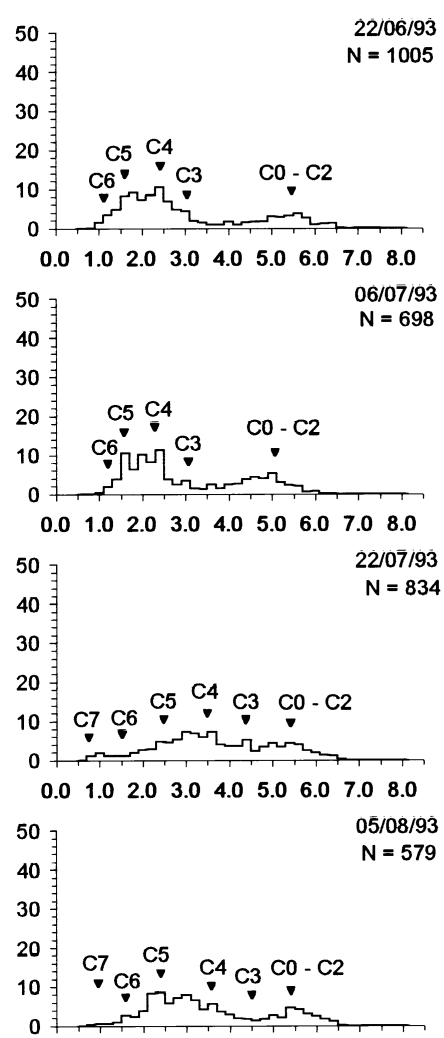

$\begin{array}{lllllllll}0.0 & 1.0 & 2.0 & 3.0 & 4.0 & 5.0 & 6.0 & 7.0 & 8.0\end{array}$

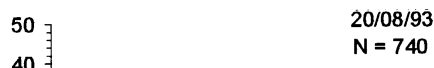

10 C7 $\quad$ C $\because \mathrm{C} 4 \mathrm{C} 3 \mathrm{CO}-\mathrm{C} 2$

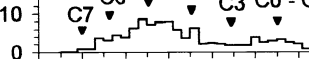
$\begin{array}{lllllllll}0.0 & 1.0 & 2.0 & 3.0 & 4.0 & 5.0 & 6.0 & 7.0 & 8.0\end{array}$

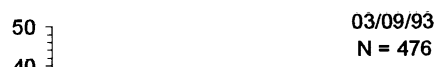

30

30

20
10
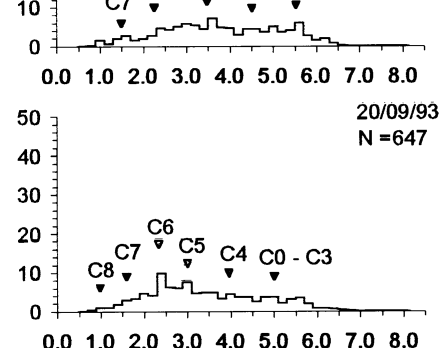

Shell height (mm)
Eutrophied area
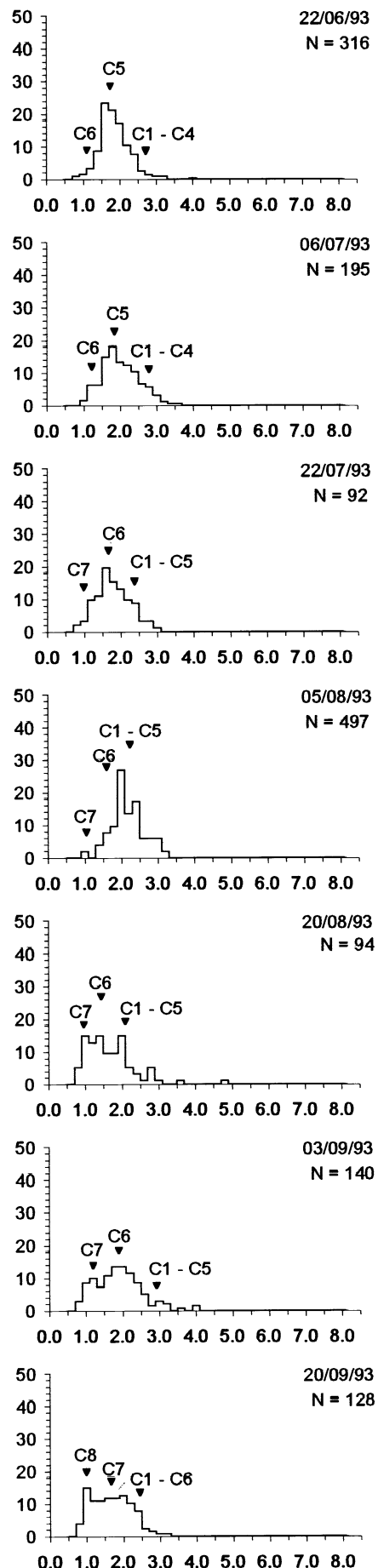

Shell height (mm)
Strongly eutrophied area
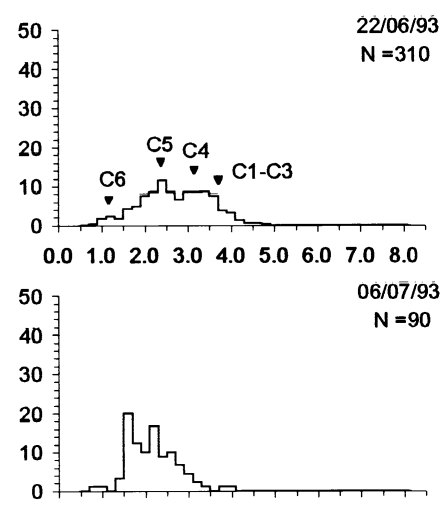

$\begin{array}{lllllllll}0.0 & 1.0 & 2.0 & 3.0 & 4.0 & 5.0 & 6.0 & 7.0 & 8.0\end{array}$

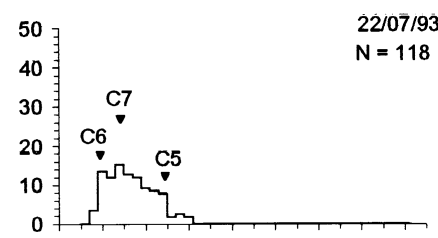

$\begin{array}{lllllllll}0.0 & 1.0 & 2.0 & 3.0 & 4.0 & 5.0 & 6.0 & 7.0 & 8.0\end{array}$

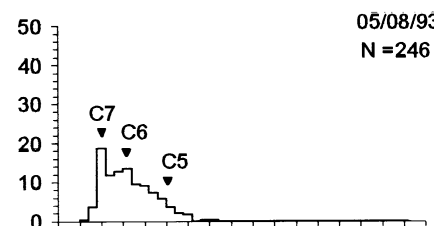

$\begin{array}{llllllllll}0.0 & 1.0 & 2.0 & 3.0 & 4.0 & 5.0 & 6.0 & 7.0 & 8.0\end{array}$

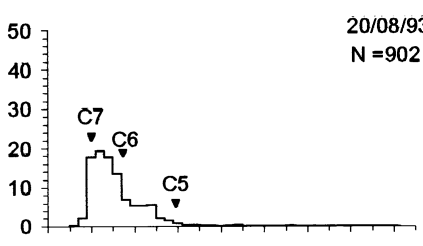

$\begin{array}{lllllllll}0.0 & 1.0 & 2.0 & 3.0 & 4.0 & 5.0 & 6.0 & 7.0 & 8.0\end{array}$
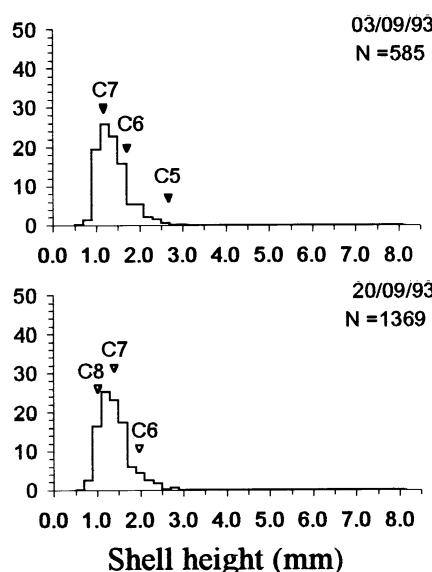

Shell height (mm)

Figure 7. (cont.) 
Zostera noltii meadows
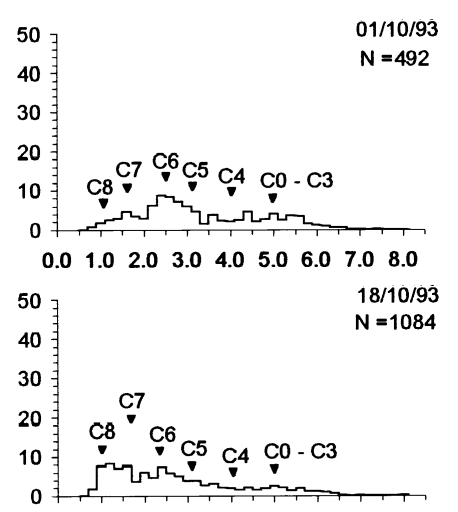

$\begin{array}{lllllllll}0.0 & 1.0 & 2.0 & 3.0 & 4.0 & 5.0 & 6.0 & 7.0 & 8.0\end{array}$

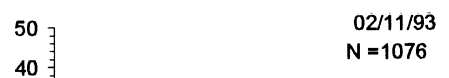

30

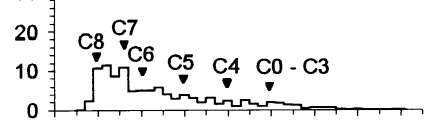

$\begin{array}{lllllllll}0.0 & 1.0 & 2.0 & 3.0 & 4.0 & 5.0 & 6.0 & 7.0 & 8.0\end{array}$

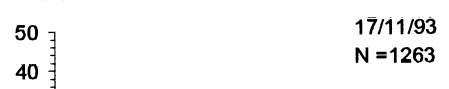

30

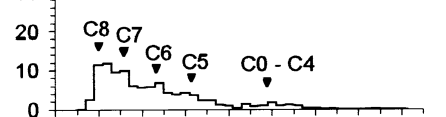

$\begin{array}{lllllllll}0.0 & 1.0 & 2.0 & 3.0 & 4.0 & 5.0 & 6.0 & 7.0 & 8.0\end{array}$
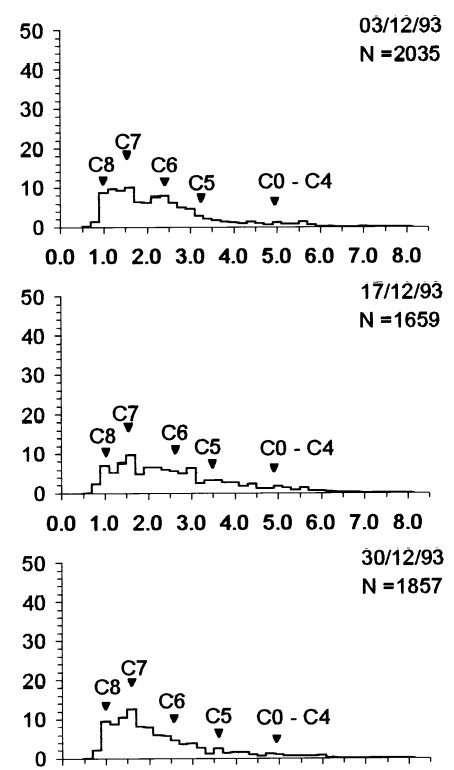

$\begin{array}{lllllllll}0.0 & 1.0 & 2.0 & 3.0 & 4.0 & 5.0 & 6.0 & 7.0 & 8.0\end{array}$

Shell height (mm)
Eutrophied area

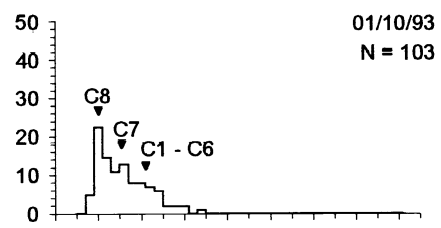

$\begin{array}{lllllllll}0.0 & 1.0 & 2.0 & 3.0 & 4.0 & 5.0 & 6.0 & 7.0 & 8.0\end{array}$
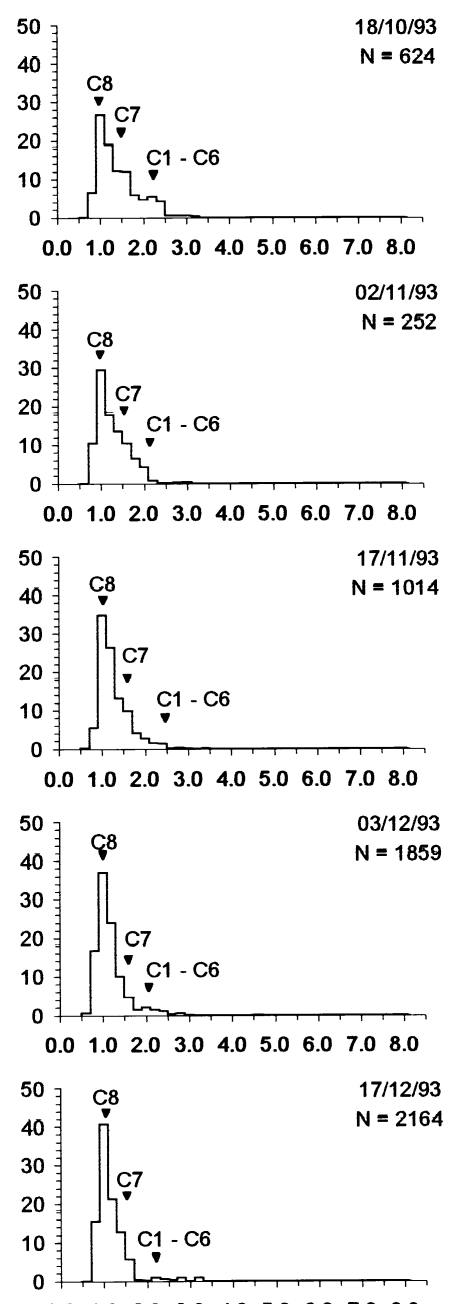

$\begin{array}{lllllllll}0.0 & 1.0 & 2.0 & 3.0 & 4.0 & 5.0 & 6.0 & 7.0 & 8.0\end{array}$
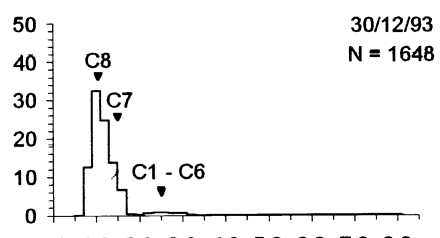

$\begin{array}{lllllllll}0.0 & 1.0 & 2.0 & 3.0 & 4.0 & 5.0 & 6.0 & 7.0 & 8.0\end{array}$

Shell height (mm)
Strongly eutrophied area

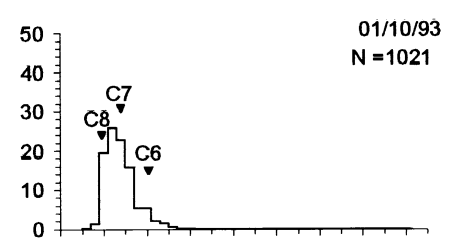

$\begin{array}{lllllllll}0.0 & 1.0 & 2.0 & 3.0 & 4.0 & 5.0 & 6.0 & 7.0 & 8.0\end{array}$
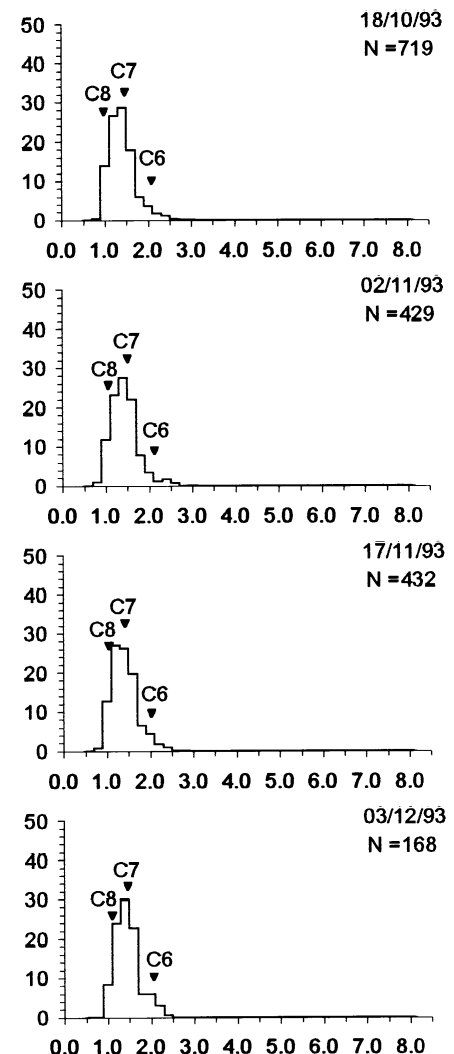

$\begin{array}{ccccccccc}0.0 & 1.0 & 2.0 & 3.0 & 4.0 & 5.0 & 6.0 & 7.0 & 8.0 \\ & & & & & & & & 17 / 12 / 93\end{array}$

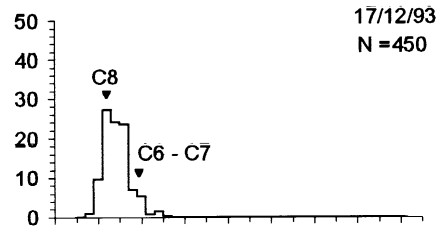

$\begin{array}{lllllllll}0.0 & 1.0 & 2.0 & 3.0 & 4.0 & 5.0 & 6.0 & 7.0 & 8.0\end{array}$

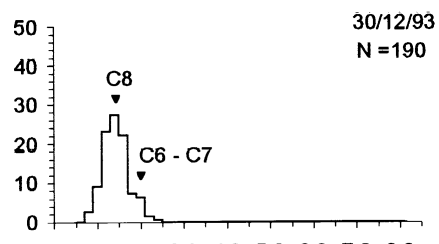

$\begin{array}{lllllllll}0.0 & 1.0 & 2.0 & 3.0 & 4.0 & 5.0 & 6.0 & 7.0 & 8.0\end{array}$

Shell height (mm)

Figure 7. (cont.) 
Zostera noltii meadows
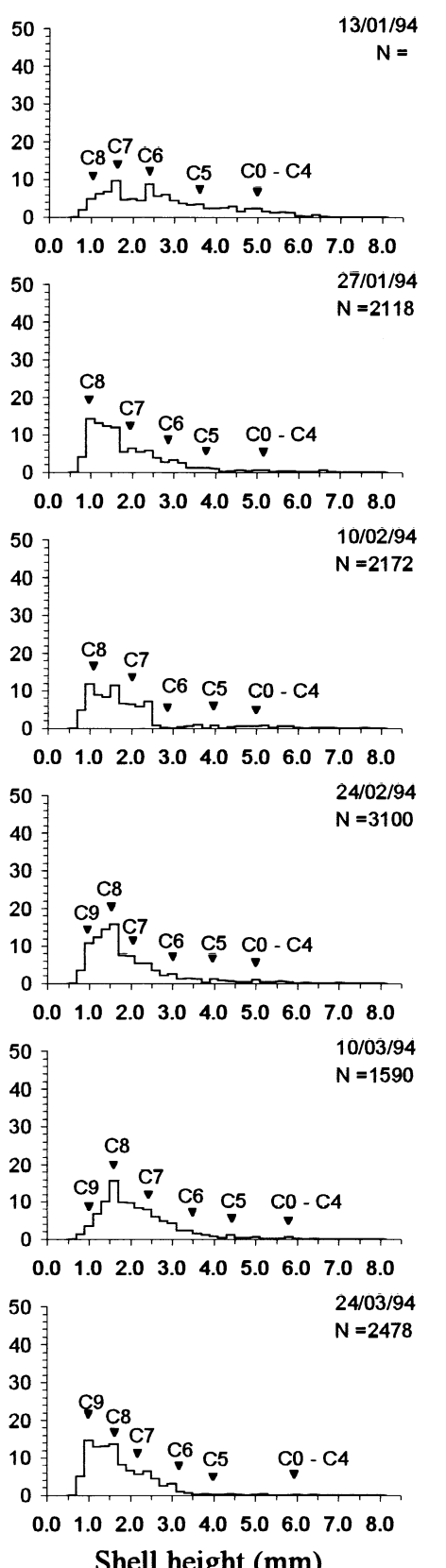

Shell height (mm)
Eutrophied area
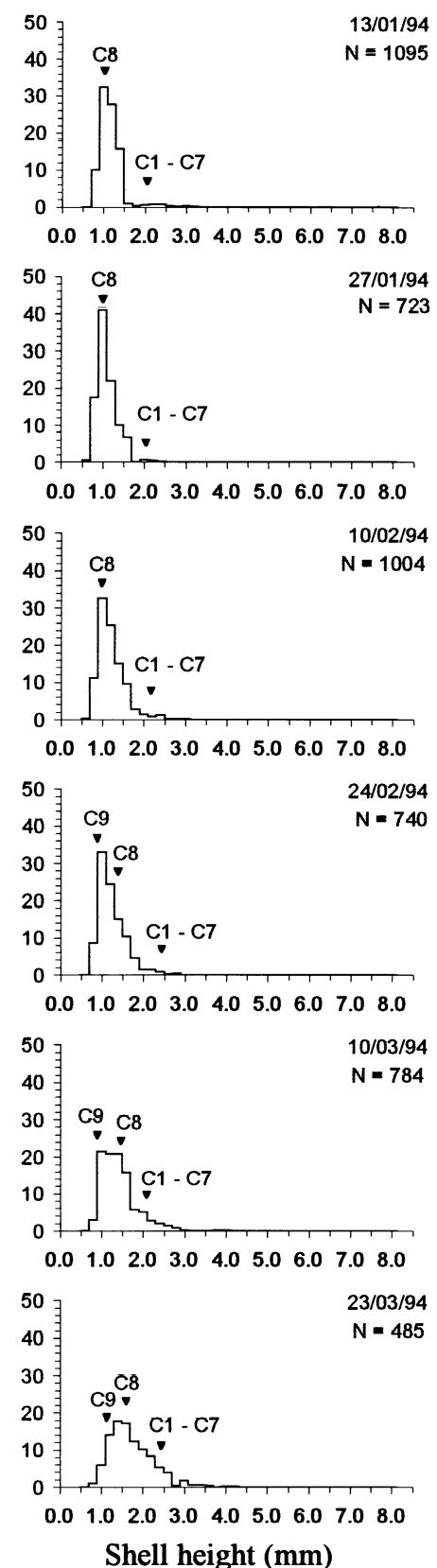

Shell height (mm)
Strongly eutrophied area
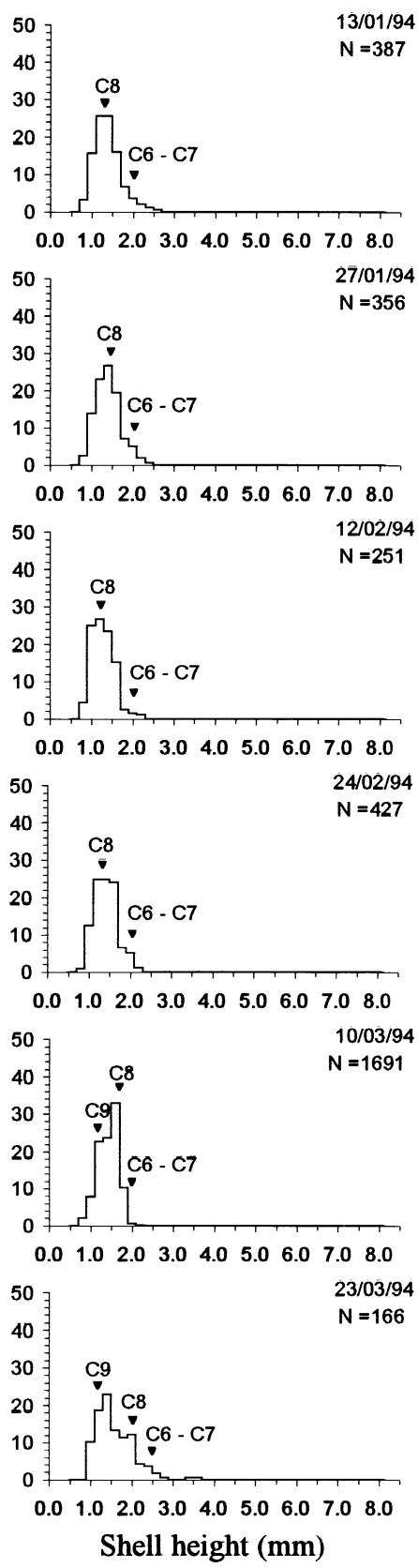

Figure 7. (cont.) 

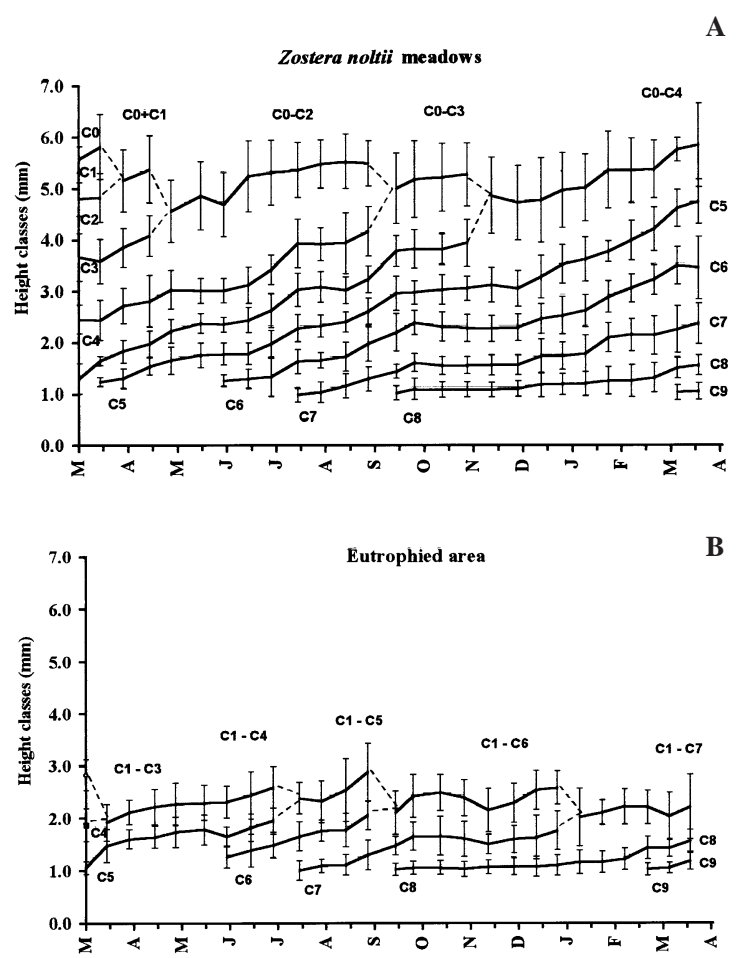

Strongly eutrophied area

C

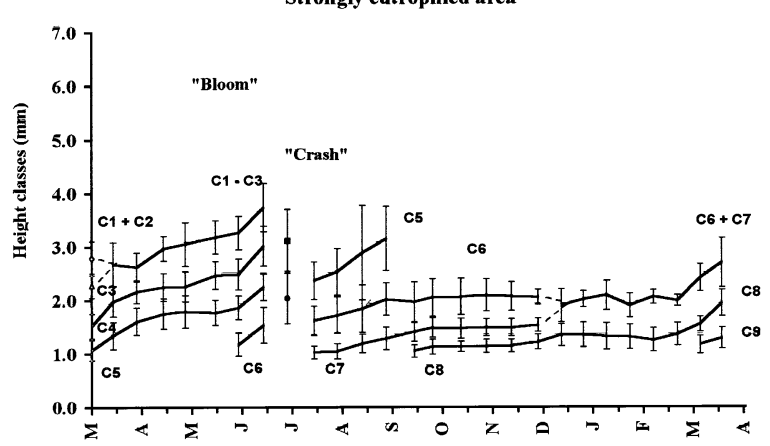

Figure 8. Estimated growth (field growth data) of Hydrobia ulvae cohorts or groups of cohorts, average total length \pm standard deviation during the study period. Broken lines indicate probable cohort merging. A, Zostera noltii meadows; B, eutrophied area; C, strongly eutrophied area.

$15.2 \mathrm{~g} \mathrm{AFDW} \cdot \mathrm{m}^{-2} \cdot \mathrm{year}^{-1}$ in the eutrophied area, and $30.3 \mathrm{~g} \mathrm{AFDW} \cdot \mathrm{m}^{-2} \cdot$ year $^{-1}$ in the strongly eutrophied area. Elimination productivity (E) was estimated at $30.0 \mathrm{~g} \mathrm{AFDW} \cdot \mathrm{m}^{-2} \cdot$ year $^{-1}$ in the $Z$. noltii meadows, $51.8 \mathrm{~g} \mathrm{AFDW} \cdot \mathrm{m}^{-2} \cdot$ year $^{-1}$ in the eutrophied area, and $97.5 \mathrm{~g} \mathrm{AFDW} \cdot \mathrm{m}^{-2} \cdot \mathrm{year}^{-1}$ in the strongly eutrophied area. The average annual biomass $(\overline{\mathrm{B}})$ (standing stock) of the population was estimated at $70.2 \mathrm{~g}$ AFDW $\cdot \mathrm{m}^{-2}$ in the $Z$. noltii meadows, $5.5 \mathrm{~g} \mathrm{AFDW} \cdot \mathrm{m}^{-2}$ in the

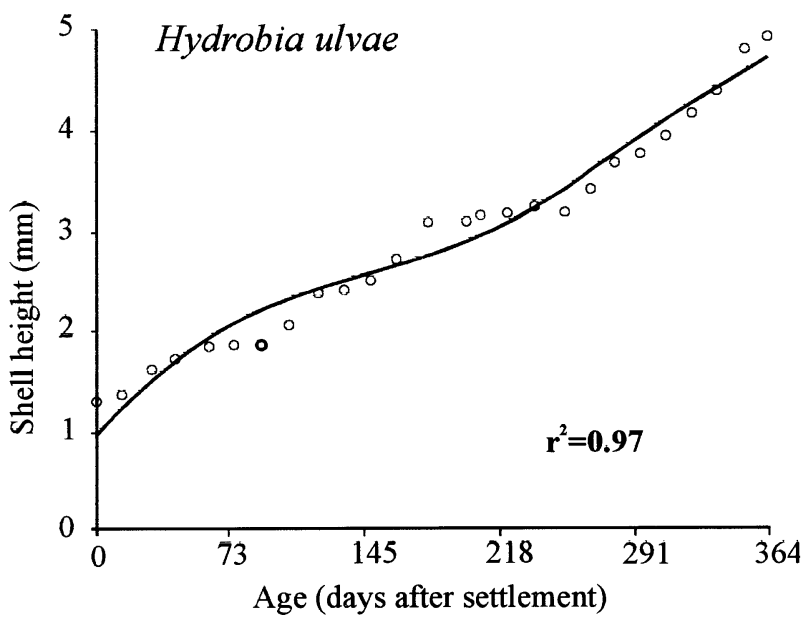

Figure 9. Hydrobia ulvae growth model for the south arm of the Mondego estuary.

eutrophied area, and $7.4 \mathrm{~g} \mathrm{AFDW} \cdot \mathrm{m}^{-2}$ in the strongly eutrophied area. $\mathrm{P} / \overline{\mathrm{B}}$ and $\mathrm{E} / \overline{\mathrm{B}}$ ratios were estimated respectively at 1.3 and 4.8 in the $Z$. noltii meadows, 2.8 and 9.5 in the eutrophied area, and 4.5 and 13.2 in the strongly eutrophied area. Taking into account the two scenarios considered at the strongly eutrophied area, we estimated $\mathrm{P} / \overline{\mathrm{B}}=5.3$ and $\mathrm{E} / \overline{\mathrm{B}}=14.4$ during the algal bloom, while after the algal crash $\mathrm{P} / \overline{\mathrm{B}}=3.3$ and $\mathrm{E} / \overline{\mathrm{B}}=11.4$.

These results demonstrate that $H$. ulvae standing stock decreased as a function of increasing eutrophication, while $\mathrm{P} / \overline{\mathrm{B}}$ and $\mathrm{E} / \overline{\mathrm{B}}$ ratios increased following the same gradient.

\section{Percentage of $\mathrm{H}$.ulvae carring egg masses}

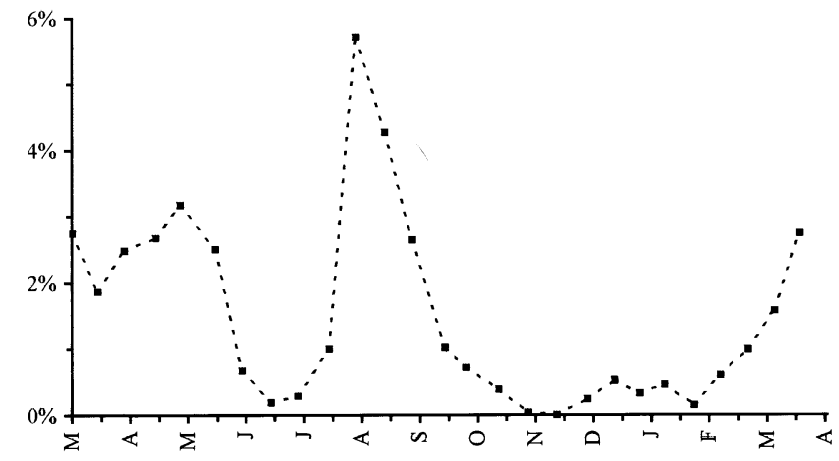

Figure 10. Percentage of Hydrobia ulvae caring egg masses in the south arm of the Mondego estuary between March 93 and March 94. 


\section{DISCUSSION AND CONCLUSIONS}

The study of $H$. ulvae population dynamics along the eutrophication gradient provided an opportunity to assess its capacity to adapt to environmental changes. Moreover, production estimates contributed to the evaluation of the role of this species with regard to energy flow in the estuarine food web.

\subsection{Spatial distribution and abundance}

Hydrobia ulvae presented a contiguous spatial distribution in the intertidal zone [14], which is consistent with results from other authors (see references in [11]).

Population density clearly changed along the eutrophication gradient, probably as a function of the vegetation dynamics. The Zostera noltii meadows presented a stable and dense $H$. ulvae population all over the study period, which is consistent with results from other authors [50], probably as a result of the greater stability provided by macrophyte beds, in comparison to green macroalgae-covered areas. Another possible explanation is the fact that this area is constituted by muddy sediments, with higher organic matter content, which may regulate distribution and abundance of $H$. ulvae [7]. Densities recorded were clearly higher than, for instance, those observed by Curras and Mora [10] and Sola [45, 46], which may be due to the fact that these authors used a $1.0-\mathrm{mm}$ mesh size sieve. In fact, this mesh size is not effective in capturing individuals smaller than $1.5 \mathrm{~mm}$ [6]. Nevertheless, Planas and Mora [43] observed a maximum abundance of $100028 \mathrm{ind} \cdot \mathrm{m}^{-2}$ in the ria de Pontevedra, Spain.

At the Z. noltii meadows, H. ulvae density increased from early winter 1993 to a maximum in spring 1994. This is not in agreement with observations carried out by other authors, e.g. Curras and Mora [10] in the ria del Eo, Spain, where the maximum density occurred in spring and summer and the minimum during winter. Nevertheless, our data may be related to the fact that no algal bloom occurred at the strongly eutrophied area of the estuary in spring 1994 [9]. As a result, the usual alternative macroalgal habitats were not available and juvenile migration [2] probably did not occur. This may explain as well the low population density observed in the strongly eutrophied area in spring 1994. In fact, there were two distinctive scenarios in the strongly eutrophied area. During the algal bloom, the population density was much higher (167 510 ind $\cdot \mathrm{m}^{-2}$ in April 7, 1993) than in the Z. noltii meadows in the same period. This may be related to habitat heterogeneity and food resources $[15,35,47$, 49]. During the algal crash, when anoxic conditions occurred in the sediment surface, the population suffered a sudden and drastic reduction, from 21818
(June 22) to 699 ind $\cdot \mathrm{m}^{-2}$ (July 6). After the algal crash, although the population was able to recover (62 863 ind $\cdot \mathrm{m}^{-2}$, August 20), it could not reach initial values again. Such recovery may have resulted from new settlements and/or the byssus-drift migration of $H$. ulvae juveniles $[2,3]$. In the present study, $H$. ulvae showed a remarkable capacity to re-colonise the disturbed area. Furthermore, other authors have reported its migration capacity $[45,46]$ and tolerance to adverse conditions $[43,45]$.

\subsection{Environmental factors}

$H$. ulvae biomass at the $Z$. noltii meadows was positively correlated with green algae biomass and negatively correlated with $Z$. noltii below-ground biomass and salinity. Although, Enteromorpha spp. and Ulva sp. biomass were very low this positive correlation can be explained by the fact that an organic enrichment may favour algal development $[15,18,21$, $23,25,30,40,44]$. In addition, grazing activity on periphyton and epiphyte may favour the macrophytes [22, 42], and therefore food resources were always available for $H$. ulvae $[16,20,24,36]$. The negative correlation with $Z$. noltii below-ground biomass may be explained by the fact that leaves and root rhizomes showed opposite peaks during the study period. Furthermore, Asmus and Asmus' [4] observations in the Wadden Sea showed that epiphytic diatoms density, which constitute a main food item for $H$. ulvae [16], always followed Z. noltii leaf biomass. The negative correlation with salinity cannot be explained as a cause-effect relationship, since $H$. ulvae tolerates a wide salinity range (10 to $35 \%$ ) [35]. This result must therefore be considered as incidental. In fact, $H$ ulvae abundance and biomass increased from winter 93 to spring 94 and as this was a very rainy period salinity values decreased, and were lower in spring 94.

At the eutrophied area and during the algal bloom, H. ulvae biomass was positively correlated with the green macroalgae biomass, with organic matter content in sediment and with oxygen concentration. While, after the algal crash, it was positively correlated with Enteromorpha spp. and Ulva sp. biomass and oxygen concentration. A positive correlation with macroalgal biomass makes sense since algae-covered habitats can provide food resources and shelter [15, 23, 50]. In fact, Drake and Arias [11] showed that algal biomass was the most important factor for hydrobiid species temporal and spatial patterns in the Bay of Cadiz, Spain. The positive correlation between the sediment organic matter contents and $H$. ulvae biomass can be related to the fact that in this area, organic matter could be a limiting factor [23]. A possible explanation for the results regarding the oxygen concentration in this area resides in the fact that, during 
the bloom, due to respiration, a huge green macroalgae biomass may have induced anoxic conditions during nocturnal periods which strongly affected the $H$. ulvae population causing its decline. As we always measured oxygen concentrations during the day, such conditions could not be detected but it was clear that $H$. ulvae biomass followed the decrease of green macroalgae biomass. In fact, Everett [15] found dissolved oxygen concentration in the algae-sediment water interface to be significantly lower than in overlying water column, and Hull [23] observed that sediments under algal mats had significantly lower redox potentials, when compared to bare bottom sediment. In addition, in the Bay of Cadiz, Drake and Arias [11] found that $H$. ulvae abundance was highest in the sites with the greatest water renewal.

\subsection{Growth, life span and productivity}

The settlement pattern of H. ulvae in the Mondego estuary was the same along the eutrophication gradient, with settlements occurring in March, June, July and September. This is not entirely coincident with results obtained by Bachelet and Yacine-Kassab [6], Curras and Mora [10] and Sola [45, 46], but differences may arise from contrasting geographic environmental conditions. Other possible explanations could be related to sampling periodicity or to sieve mesh size (we used 500- $\mu \mathrm{m}$, while other authors used $1000 \mu \mathrm{m}$ which may cause a certain bias) or even to the methodology applied in cohorts detection. Nevertheless, it is possible to establish some correspondence between the settlements taking into account the mean shell height of the cohorts.

Two main reproductive peaks, but very small when compared two other authors' [11, 17], were recognisable in the Mondego estuary population. Although, this result may not be consistent with the settlement pattern of $H$. ulvae, only a very small proportion of egg capsules was observed on snail shells. In the Mondego estuary, $H$. ulvae egg capsules were mainly observed on macroalgae and Z. noltii leaves, but they were not quantified. Nevertheless, contrasting with the scarcity of egg capsules, $H$. ulvae abundance and population structure show a successful reproduction and recruitment of this species.

Through cohort recognition and tracking, it was possible to assess growth and to estimate life span at $21 \pm 3$ months. The estimate for the Mondego estuary population was similar to the estimate for the Medway estuary, UK [50]. But slightly higher than for the ria de Pondevedra, Spain [43], and for the ria del Eo, Spain [10], and lower than estimates for the Dovey estuary, UK [17], and for the Gironde estuary, France [6].
The $H$. ulvae standing stock decreased as a function of increasing eutrophication, while $\mathrm{P} / \overline{\mathrm{B}}$ and $\mathrm{E} / \overline{\mathrm{B}}$ ratios increased following the same gradient. This was obviously related with the size of individuals found in each sampling area, in relation with the dynamics of macroalgal mats. Probably as a result from higher exposure to predators [37], $H$. ulvae individuals were considerably smaller than in the $Z$. noltii meadows. Moreover, these results may suggest that $H$. ulvae might be suffering a change in its adaptive strategy along the eutrophication gradient, becoming closer to a ' $r$ ' strategy.

The H. ulvae $\mathrm{P} / \overline{\mathrm{B}}$ estimates in the Mondego estuary were of the same order of magnitude as estimates for the same species in similar habitats, e.g. algae-covered areas [11] and Z. noltii meadows [48]. Exception is made for the strongly eutrophied area where these estimates were higher. In this area, and considering the two distinctive situations, $\mathrm{P} / \overline{\mathrm{B}}$ and $\mathrm{E} / \overline{\mathrm{B}}$ estimates reflect that under these unstable environmental conditions, exposure to anoxic conditions during the algal crash and to predators, $H$. ulvae population structure is altered probably as a function of life expectancy.

In the Mondego estuary, $H$. ulvae production was very high when compared to Ria Formosa, Portugal [48] and also when compared with estimates of secondary production of whole intertidal benthic communities [48]. Nevertheless, $H$. ulvae $\mathrm{P} / \overline{\mathrm{B}}$ in the $Z$. noltii meadow can be compared to Sprung's [48] estimate for the whole intertidal benthic communities, taking into account the fact that $H$. ulvae represents more than $80 \%$ of the total benthic macrofauna biomass in the Mondego estuary [40].

The present results reinforce the generalised notion that estuaries are highly productive systems, and that $H$. ulvae may be seen as an important element of this energy flow. Moreover, evidence is shown that $H$. ulvae population structure and annual production were affected by eutrophication, namely by macroalgal bloom dynamics.

\section{Acknowledgments}

The present work was supported by the programme MAST II (MAS 2 CT 92 0036) and by JNICT (Portuguese National Institution for Scientific Research) by conceding a M.Sc. grant (BM/ 1760/ 94) in the scope of the Praxis XXI programme. A very special thanks goes to all the colleagues from IMAR-Coimbra.

\section{REFERENCES}

[1] Allen K.R., Relation between production and biomass, J. Fish. Res. Board Can. 28 (1971) 1573-1581.

[2] Armonies W., Migratory rhythms of drifting juvenile molluscs in tidal waters of the Wadden Sea, Mar. Ecol. Prog. Ser. 83 (1992) 197-206. 
[3] Armonies W., Hartke D., Floating of mud snail Hydrobia ulvae in tidal waters of the Wadden Sea, and its implications in distribution patterns, Helgol. Meeresunters 49 (1995) 529-538.

[4] Asmus H., Asmus R., The importance of grazing food chain for energy flow and production in three intertidal sand bottom communities of the northern Wadden Sea., Helgol. Meeresunters 39 (1985) 273-301.

[5] Azeiteiro U.M.M., Marques J.C., Temporal and spatial structure in the suprabenthic community of a shallow estuary (western Portugal: Mondego river estuary), Acta Oecol. 20 (1999) 333-342.

[6] Bachelet G., Yacine-Kassab M., Intégration de la phase post-recrutée dans la dynamique des populations du gastéropode intertidal Hydrobia ulvae (Pennant), J. Exp. Mar. Biol. Ecol. 111 (1987) 37-60.

[7] Barnes R.S.K., Intrapopulation variation in Hydrobia sediment preferences, Estuar. Coast. Mar. Sci. 9 (1979) 231-234.

[8] Brown A.C., McLachan A., Wentworth size scale for sediments, in: Ecology of Sandy Shores, Elsevier, 1990, 328 p.

[9] Cabral J.A., Pardal M.A., Lopes R.J., Múrias T., Marques J.C., The impact of macroalgal blooms on the use of the intertidal area and feeding behaviour of waders (Charadrii) in the Mondego estuary (west Portugal), Acta Oecol. 20 (1999) 417-427.

[10] Curras A., Mora J., Dinamica poblacional de Bittium reticulatum (Da Costa) e Hydrobia ulvae (Pennant) en fondos de Zostera spp. de la ria del Eo (Galcia-Asturias), Thalassas 8 (1990) 93-99.

[11] Drake P., Arias A.M., Distribution and production of three Hydrobia species (Gastropoda: Hydrobiidae) in a shallow coastal lagoon in the Bay of Cádiz (Spain), J. Molluscan Stud. 61 (1995) 185-196.

[12] Edwards A.L., An Introduction to Linear Regression and Correlation, 2nd ed., W.H. Freeman \& Company, New York, 1984, 206 p.

[13] Edwards A.L., Multiple Regression and Analysis of Variance and Covariance, 2nd ed., W.H. Freeman \& Company, New York, 1985, $221 \mathrm{p}$.

[14] Elliot J.M., Statistical Analysis of Samples of Benthic Invertebrates, 2nd ed., Freshwater Biological Association, Scientif. Publ. No. 25, 1977, 160 p.

[15] Everett R.A., Macroalgae in marine soft-sediment communities: Effects on benthic faunal assemblages, J. Exp. Mar. Ecol. 175 (1994) 253-274.

[16] Fenchel T., Kofoed L.H., Lappalainen A., Particle size selection of deposit feeders: the amphipod Corophium volutator and the prosobranch Hydrobia ulvae, Mar. Biol. 30 (1975) 119-128.

[17] Fish J.D., Fish S., The breeding cycle and growth of Hydrobia ulvae in the Dovey estuary, J. Mar. Biol. Assoc. UK 54 (1974) 685-697.

[18] Flindt M.R., Kamp-Nielsen L., Marques J.C., Pardal M.A., Bocci M., Bendoricho G., Nielsen S.N., Jørgensen S.E., Description of the three shallow estuaries: Mondego River (Portugal), Roskilde Fjord (Denmark) and the Lagoon of Venice (Italy), Ecol. Model. 102 (1997) 17-31.

[19] Gaschütz G., Pauly D., David N., A versatile basic program for fitting weight and seasonally oscillating length growth data, Pelagic, Demersal and Shellfish Cttes, 1980, 23 p.
[20] Graham A.F.R.S., Molluscs: prosobranch and pyramidellid gastropods, in: Kermack D.M., Barnes R.S.K. (Eds.), Synopses of the British Fauna (New Series), 2nd ed., London, No. 2, 1988, 662 p.

[21] Hardy F.G., Evans S.M., Tremayne M.A., Long-term changes in the marine macroalgae of three polluted estuaries in north-east England, J. Exp. Biol. Ecol. 172 (1993) 82-92.

[22] Howard R.K., Short F.T., Seagrass growth and survivorship under the influence of epiphytes grazers, Aquat. Bot. 24 (1986) 287-302.

[23] Hull S.C., Macroalgal mats and species abundance: a field experiment, Estuar. Coast. Shelf Sci. 25 (1987) 519-532.

[24] Jensen K.T., Siegismund H.R., The importance of diatoms and bacteria in the diet of Hydrobia species, Ophelia 1 (Suppl.) (1980) 193-199.

[25] Lavery P.S., Lukatelich R.J., McComb A.J., Changes in the biomass and species composition of macroalgae in a eutrophic estuary, Estuar. Coast. Shelf Sci. 33 (1991) 1-22.

[26] Lillebø A.I., Estrutura populacional, dinâmica e produção de Hydrobia ulvae (Pennant) (Mollusca:Prosobranchia) ao longo de um gradiente de eutrofização do estuário do Mondego, M.Sc. thesis, University of Coimbra, Portugal, 1996, 111 p.

[27] Lilleb $\varnothing$ A.I., Flindt M.R., Pardal M.A., Marques J.C., The effect of macrofauna, meiofauna and microfauna on the degradation of Spartina maritima detritus from a salt marsh area, Acta Oecol. 20 (1999) 249-258.

[28] Marques J.C., Amphipoda (Crustacea) bentónicos da costa portuguesa: estudo taxonómico, ecológico e biogeográfico, Ph.D. thesis, University of Coimbra, Portugal, 1989, 394 p.

[29] Marques J.C., Maranhão P., Pardal M.A., Human impact assessment on the subtidal macrobenthic community structure in the Mondego estuary (western Portugal), Estuar. Coast. Shelf Sci. 37 (1993) 403-419.

[30] Marques J.C., Rodrigues L.B., Nogueira J.A., Intertidal macrobenthic communities structure in the Mondego estuary (western Portugal), Vie Milieu 43 (1993) 177-187.

[31] Marques J.C., Martins I., Teles-Ferreira C., Cruz S., Population dynamics, life history, and production of Cyathura carinata (Krøyer) (Isopoda: Anthuridae) in the Mondego estuary, Portugal, J. Crustac. Biol. 14 (1994) 258-272.

[32] Marques J.C., Pardal M.A., Nilsen S.N., Jørgensen S.E., Analysis of the properties of exergy and biodiversity along an estuarine gradient of eutrophication, Ecol. Model. 62 (1997) 155-167.

[33] Martins I.I.C., Modelling the effects of green macroalgae blooms on the population dynamics of Cyatura carinata (Crustacea: Isopoda) in the Mondego estuary (Portugal), M.Sc. thesis, University of Coimbra, Portugal, 1995, $131 \mathrm{p}$.

[34] Martins I., Oliveira J.M., Flindt M.R., Marques J.C., The effect of salinity on the growth rate of the macroalgae Enteromorpha intestinalis (Chlorophyta) in the Mondego estuary (west Portugal), Acta Oecol. 20 (1999) 259-265.

[35] McLusky D.S., The Estuarine Ecosystem, Blakie \& Son, Glasgow and London, 1988, 250 p.

[36] Morrisey D.J., Differences in effects of grazing by depositfeeders Hydrobia ulvae (Pennant) (Gastropoda: Prosobranchia) and Corophium arenarium (Amphipoda) on sediment microalgal populations. II. Quantitative effects, J. Exp. Mar. Biol. Ecol. 118 (1988) 43-53. 
[37] Múrias T., Cabral J.A., Marques J.C., Goss-Custard J.D., Short-term effects of intertidal macroalgal blooms on the macrohabitat selection and feeding behaviour of wading birds in the Mondego estuary (West Portugal), Estuar. Coast. Shelf Sci. 43 (1996) 677-688.

[38] Nogueira A.J., ANAMOD - Extracção dos componentes modais de distribuições de frequências de variáveis biométricas, Provas de Aptidão Pedagógica e Capacidade Científica, University of Coimbra, Portugal, 1992, 67 p.

[39] Pardal M.A., Variação espacial e temporal das populações de poliquetas no estuário do Mondego. Dinâmica e produção de Amage adspersa (Grube, 1863), M.Sc. thesis, University of Coimbra, Portugal, 1995, $121 \mathrm{p}$.

[40] Pardal M.A., Impacto da eutrofização nas comunidades macrobentónicas do braço sul do estuário do Mondego (Portugal), Ph.D. thesis, University of Coimbra, Portugal, 1998, 315 p.

[41] Pardal M.A., Marques J.C., Bellan G., Spatial ditribution and seasonal variation of subtidal polychaete populations in the Mondego estuary (western Portugal), Cah. Biol. Mar. 34 (1993) 497-512.

[42] Philippart C.J.M., Effect of periphyton grazing by Hydrobia ulvae on the growth of Zostera noltii on a tidal flat in the Dutch Wadden Sea, Mar. Biol. 122 (1995) 431-437.
[43] Planas M., Mora J., Dinámica poblacional de Hydrobia ulvae (Pennant) en la ria de Pontevedra (NO Espanã), Cah. Biol. Mar. 28 (1987) 447-464.

[44] Shories D., Reise K., Germination and anchorange of Enteromorpha spp. in sediments of the Wadden Sea, Helgol. Meeresunters 47 (1993) 275-285.

[45] Sola J.C.S., Estudio de la communidad reducida de Macoma en el estuario del Bidasoa, Ph.D. thesis, Servicio Editorial Universidad del Pais Vasco, 1994, 526 p.

[46] Sola J.C.S., Population dynamics, reproduction, growth, and secondary production of the mud-snail Hydrobia ulvae (Pennant), J. Exp. Mar. Biol. Ecol. 205 (1996) 49-62.

[47] Soulsby P.G., Lowthion D., Houston M., Effects on macroalgae mats on the ecology of intertidal mudflats, Mar. Pollut. Bull. 13 (1982) 162-166.

[48] Sprung M., Macrobenthic secondary production in the intertidal zone of the Ria Formosa - a lagoon in southern Portugal, Estuar. Coast. Shelf Sci. 38 (1994) 539-558.

[49] Vadas R.L., Beal B., Green algal ropes: A novel estuarine phenomenon in the Gulf of Maine, Estuaries 10 (1987) 171-176.

[50] Walters G.J., Wharfe J.R., Distribution and abundance of Hydrobia ulvae (Pennant) in the lower Medway estuary, Kent, J. Molluscan Stud. 46 (1980) 171-180. 